\title{
DEFORMATIONS AND MOMENTS OF INERTIA OF ACTINIDE NUCLEI IN THE GROUND AND SHAPE ISOMERIC STATES
}

\author{
M. BRACK ${ }^{\dagger}$, T. LEDERGERBER ${ }^{\dagger \dagger}$ and H. C. PAULI ${ }^{+{ }^{+}}$ \\ Institute for Theoretical Physics, Basel. Schweiz \\ and \\ A. S. JENSEN \\ Institute of Physics, University of Aarhus, Denmark \\ Received 31 May 1974
}

\begin{abstract}
Using a Woods-Saxon potential, equilibrium deformations are obtained by the Strutinsky shell-correction method. Deformation parameters $\beta_{2}$ and $\beta_{4}$ of the ground state and the shape isomeric state are extracted for all actinide nuclei. It is shown that the connection of $\beta_{2}$ and $\beta_{4}$ with the multipole moments $Q_{2}$ and $Q_{4}$ is not so trivial as sometimes assumed in the literature. The moments of inertia - taken at the same deformations - are evaluated within the cranking model. Their dependence on deformation and temperature (excitation energy) is discussed; the rigid body values are demonstrated to be reached both for large deformations and large tem. peratures. Where available, experimental data are compared; the agreement is generally very good.
\end{abstract}

\section{Introduction}

The experimental information on the actinide nuclei is increasing. Ground-state quadrupole and hexadecapole moments of twelve actinide nuclei were recently measured ${ }^{1}$ ). Rotational spectra built on the lowest $0^{+}$state of two fission isomers have been observed ${ }^{2,3}$ ). The moments of inertia which are an indirect estimate of the deformations, are deduced. There is some hope for a more direct measurement of the quadrupole moment in the isomeric state ${ }^{4}$ ). Therefore, it seems useful to provide experimentalists with systematic tables of deformations and moments of inertia for the actinide region. Our calculations are an extension of those done by Götz et al. ${ }^{5}$ ), who restricled themselves to the ground states in the rare earth region.

The shell-correction approach of Strutinsky has provided an economical method to calculate deformation energy surfaces of nuclei ${ }^{6,7}$ ). The lowest local minimum of the surface of a given nucleus is identified with its ground state, and the next higher local minimum with its (fission) isomeric state. Both these minima are stable with respect to left-right ${ }^{8,9}$ ) and axial ${ }^{10,11}$ ) asymmetry of the shape. Thus in this paper we restrict ourselves to axial and left-right symmetric shapes, i.e. to an elongation $(c)$

† Present address: Niels Bohr Institut, Blegdamsvej 17, 2100 Copenhagen, Denmark.

tt Present address: The Weizmann Institute of Science, Dept. of Nuclear Physics, Rehovot, Israel. t†† Present address: Max-Planck-Institut für Kernphysik, Heidelberg, Germany. 
and a neck formation $(h)$ parameter. For details of the shape parametrisation we refer to refs. ${ }^{7,12}$ ).

The shell model eigenvalues, to which we apply the shell-correction method, are calculated with an average potential of the Woods-Saxon type with a constant and deformation-independent skin thickness ${ }^{7,12}$ ). Adding these shell corrections to the liquid drop energy, whose parameters are given by Pauli and Ledergerber ${ }^{13}$ ), we obtain the total deformation energy. By minimizing this energy we find the corresponding equilibrium deformation parameters $(c, h)$. Finally we calculate the quadrupole $\left(Q_{2}\right)$ and hexadecapole moments $\left(Q_{4}\right)$ of the proton and neutron density distributions of these deformations. We discuss in subsect. 2.1 the connection between the moments $Q_{2}, Q_{4}$ and the deformation of the potential. We show that care should be taken in relating the moments $Q_{2}, Q_{4}$ of the nucleon distributions to parameters $\beta_{2}, \beta_{4}$ extracted from the deformation of the potential.

The single-particle wave functions and energies at the two minima are furthermore used for calculating the moments of inertia by the cranking model ${ }^{14}$ ). Special interest is paid to the dependence of the moments of inertia on the pairing interaction strength and on the temperature of excited nuclei. These dependences are discussed and illustrated in subsect. 2.2.

A compilation of the results is presented in sect. 3 in the form of large tables. The results are compared to the available experimental data and a nice agreement is found.

\section{Discussion of qualitative features of the nuclear moments}

The shell-correction calculations with a deformed Woods-Saxon potential and the definition of the nuclear shape in terms of an elongation $(c)$ and a neck parameter $(h)$ have been described in detail ${ }^{7,12}$ ) and need not be repeated here. All single-particle wave functions and energies are calculated with parameters appropriate for ${ }^{240} \mathrm{Pu}$;

TABLE 1

Woods-Saxon parameters for ${ }^{240} \mathrm{Pu}$ (the same as quoted in ref. ${ }^{12}$ ))

\begin{tabular}{|c|c|c|c|c|}
\hline & \multicolumn{2}{|c|}{ Proton } & \multicolumn{2}{|c|}{ Neutron } \\
\hline & central & spin-orbit & central & spin-orbit \\
\hline$V_{0}(\mathrm{MeV})$ & -62.54 & 12.0 & -47.46 & 12.0 \\
\hline$R_{\mathrm{o}}(\mathrm{fm})$ & 7.79 & 7.06 & 7.73 & 7.06 \\
\hline$a(\mathrm{fm})$ & 0.66 & 0.55 & 0.66 & 0.55 \\
\hline
\end{tabular}

the potential energy surfaces of all other actinide nuclei are then obtained by $A^{\frac{1}{3}}$ scaling of the single-particle levels ${ }^{7}$ ). In table 1 we give the Woods-Saxon potential parameters of ${ }^{240} \mathrm{Pu}$ used in the present calculations; they are the same as in ref. ${ }^{12}$ ).

The pairing interaction is of special importance for the quantities considered here. For the moments of inertia, we use the temperature dependent BCS formalism for 
which we refer to refs. ${ }^{15,16}$ ). The pairing strength $G$ is given by ${ }^{7}$ )

$$
G=\left[\tilde{g}(\lambda) \ln \left(\frac{2 \Omega}{\tilde{\Delta}}\right)\right]^{-1},
$$

where the average level density $\tilde{g}(\lambda)$ at the Fermi energy (different for neutrons and protons) can be obtained from the energy spectrum by the Strutinsky averaging procedure ${ }^{6}$ ). The average pairing gap $\tilde{\Delta}$ and the energy interval $\Omega$ depend on the mass number $A$ as

$$
\begin{gathered}
\tilde{\Delta}=\frac{c_{\tau}}{\sqrt{A}} \quad(\tau=\mathrm{n}, \mathrm{p}), \\
\Omega=1.1 \hbar \omega=45 \mathrm{MeV} / A^{\frac{1}{3}},
\end{gathered}
$$

where we chose the constant $c_{\tau}=12 \mathrm{MeV}$ for both neutrons and protons as in previous calculations ${ }^{7}$ ).

Since $\tilde{g}(\lambda)$ is almost independent of the nuclear deformation, through eq. (1) also the pairing strength $G$ is essentially constant. It has been argued ${ }^{17}$ ), however, that $G$ should be proportional to the nuclear surface area $S$. Such a dependence can easily be obtained in our treatment replacing $\tilde{A}$ in eq. (1) by

$$
\tilde{\Delta}_{\mathrm{s}}=2 \Omega\left(\frac{\tilde{\Delta}_{\mathrm{o}}}{2 \Omega}\right)^{\mathrm{s}_{\mathrm{o}} / \mathrm{s}} \text {, }
$$

where $\tilde{\Delta}_{0}$ is given as in eq. (2) and $S_{0}$ is the surface area of the spherical nucleus.
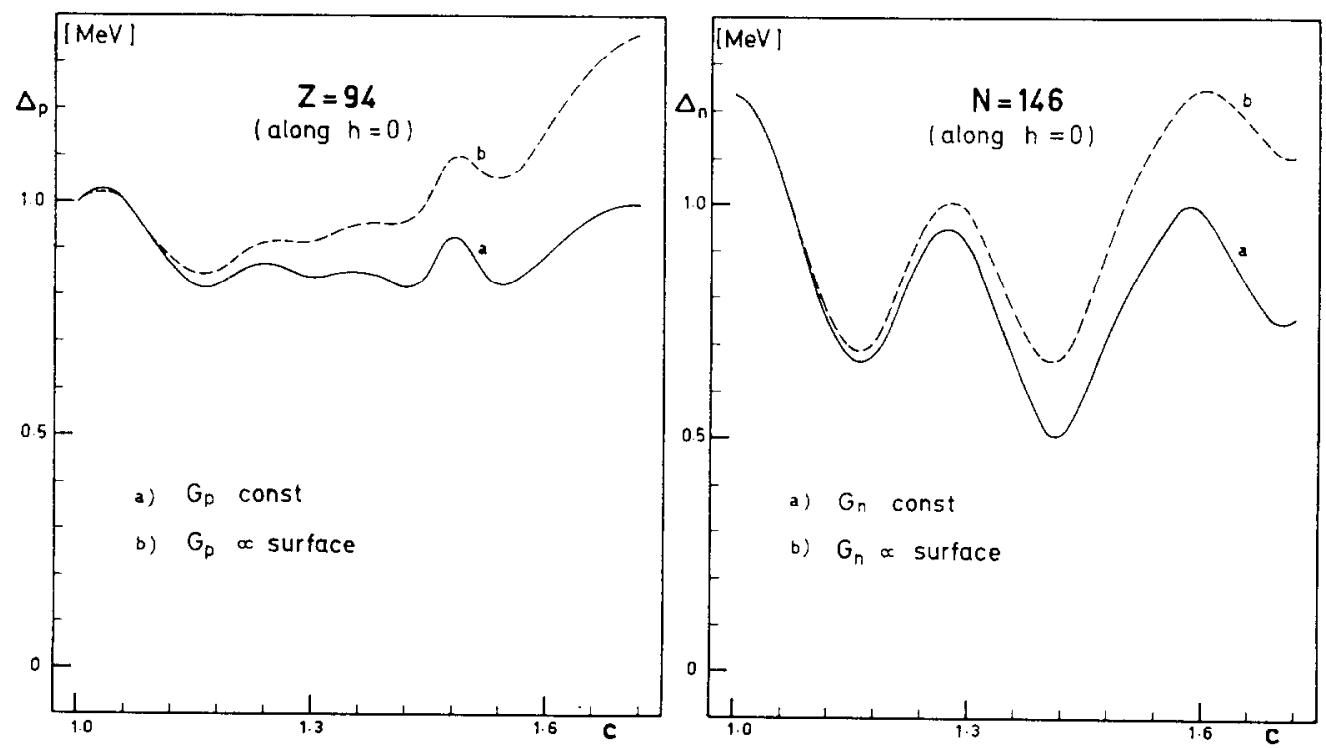

Fig. 1. Proton and neutron pairing gaps $\Delta_{\mathrm{p}}$ and $A_{\mathrm{n}}$ as functions of the elongation parameter $c$. Both constant (solid lines) and surface-dependent (dashed lines) pairing strengths $G$ are considered. 
In order to demonstrate the effect of this surface dependence on the pairing gaps, we show in fig. 1 for ${ }^{240} \mathrm{Pu}$ the gaps $\Delta_{\mathrm{n}}$ and $\Delta_{\mathrm{p}}$ as functions of the deformation parameter $c$ (along $h=0$ ). The shell structure in the local density is clearly reflected; especially in $\Delta_{\mathrm{n}}$ we see the ground-state minimum $(c \approx 1.2)$ and the isomer minimum $(c \approx 1.4)$. The difference between the two cases $G=$ constant, $G \propto S$ incrcases with deformation. While it is negligible in the ground-state region $(c \approx 1.2)$ and about $15 \%$ at the second minimum $(c \approx 1.4)$, it amounts to around $30 \%$ at the outer fission barrier $(c \approx 1.6)$. The influence of this increase on the moments of inertia will be discussed in subsect. 2.2 below.

\section{TABLE 2}

Expectation values calculated from the wave functions for some nuclei at the ground-state deformations and the second (isomeric) minima; the latter cases are marked by an asterisk

\begin{tabular}{lcccccccc}
\hline Nucleus & $r_{\mathrm{n}}{ }^{\text {rms }}$ & $r_{\mathrm{p}}{ }^{\mathrm{rms}}$ & $q_{\mathrm{n}}$ & $q_{\mathrm{p}}$ & $Q_{2}{ }^{\mathrm{n}} / N$ & $Q_{2}{ }^{\mathrm{p}} / Z$ & $Q_{4}{ }^{\mathrm{n}} / N$ & $Q_{4}{ }^{\mathrm{p}} / Z$ \\
\hline${ }^{230} \mathrm{Th}$ & 5.85 & 5.69 & 1.22 & 1.25 & 9.7 & 10.3 & 229 & 278 \\
${ }^{232} \mathrm{Th}$ & 5.88 & 5.72 & 1.24 & 1.27 & 10.6 & 11.2 & 237 & 292 \\
${ }^{234} \mathrm{U}$ & 5.88 & 5.74 & 1.24 & 1.28 & 10.6 & 11.4 & 237 & 302 \\
${ }^{236} \mathrm{U}$ & 5.91 & 5.76 & 1.26 & 1.29 & 11.3 & 12.2 & 232 & 299 \\
${ }^{238} \mathrm{U}$ & 5.94 & 5.78 & 1.26 & 1.30 & 11.6 & 12.4 & 207 & 277 \\
${ }^{238} \mathrm{Pu}$ & 5.91 & 5.79 & 1.26 & 1.30 & 11.4 & 12.5 & 219 & 283 \\
${ }^{240} \mathrm{Pu}$ & 5.96 & 5.81 & 1.27 & 1.30 & 11.7 & 12.7 & 201 & 269 \\
${ }^{242} \mathrm{Pu}$ & 5.96 & 5.82 & 1.26 & 1.30 & 11.7 & 12.7 & 158 & 228 \\
${ }^{244} \mathrm{Pu}$ & 5.98 & 5.83 & 1.26 & 1.30 & 11.8 & 12.9 & 134 & 213 \\
${ }^{244} \mathrm{Cm}$ & 5.96 & 5.84 & 1.27 & 1.30 & 11.8 & 12.9 & 143 & 212 \\
${ }^{246} \mathrm{Cm}$ & 5.99 & 5.85 & 1.27 & 1.30 & 11.9 & 12.9 & 135 & 214 \\
${ }^{248} \mathrm{Cm}$ & 6.02 & 5.87 & 1.27 & 1.31 & 12.3 & 13.5 & 126 & 216 \\
${ }^{232} \mathrm{Th}$ & 6.31 & 6.26 & 1.79 & 1.94 & 33.7 & 37.7 & 107 & 131 \\
${ }^{236} \mathrm{U}^{*}$ & 6.38 & 6.34 & 1.81 & 1.97 & 35.2 & 39.2 & 113 & 133 \\
${ }^{236} \mathrm{Pu}^{*}$ & 6.34 & 6.35 & 1.80 & 1.95 & 34.3 & 39.0 & 105 & 128 \\
${ }^{238} \mathrm{Pu}^{*}$ & 6.39 & 6.38 & 1.82 & 1.97 & 35.1 & 40.0 & 112 & 133 \\
${ }^{240} \mathrm{Pu}^{*}$ & 6.43 & 6.40 & 1.83 & 1.99 & 36.3 & 40.6 & 118 & 138 \\
${ }^{246} \mathrm{Cm}^{*}$ & 6.53 & 6.51 & 1.86 & 2.04 & 38.5 & 43.2 & 130 & 153 \\
${ }^{254} \mathrm{Fm}^{*}$ & 6.47 & 6.45 & 1.75 & 1.91 & 34.2 & 38.7 & 99 & 120 \\
& & & & & & & & \\
\hline
\end{tabular}

Ims radii in fm, axis ratios $q_{\mathrm{n}}$ and $q_{\mathrm{p}}$ as defined in eq. (6), reduced quadrupole moments in $\mathrm{fm}^{2}$ and hexadecapole moments in $\mathrm{fm}^{4}$.

\subsection{THE MULTIPOLE MOMENTS AT THE EQUILIBRIUM DEFORMATIONS}

The multipole moments $Q_{\lambda}$ and the rms radius $r_{\text {rms }}$ of an arbitrary density distribution $\rho(\mathbf{r})$ are defined as

$$
\begin{gathered}
Q_{\lambda}=\sqrt{\frac{16 \pi}{2 \lambda+1}} \int \rho(\boldsymbol{r}) r^{\lambda} Y_{\lambda 0}(\vartheta) \mathrm{d} \tau \\
r_{\mathrm{rms}}=\sqrt{\left\langle r^{2}\right\rangle}=\left[\int \rho(\boldsymbol{r}) r^{2} \mathrm{~d} \tau / \int \rho(\boldsymbol{r}) \mathrm{d} \tau\right]^{\frac{1}{2}} .
\end{gathered}
$$


We also define a generalized axis ratio $q$,

$$
q=\left[2 \int \rho(\boldsymbol{r}) z^{2} \mathrm{~d} \tau / \int \rho(\boldsymbol{r})\left(x^{2}+y^{2}\right) \mathrm{d} \tau\right]^{\frac{1}{2}},
$$

where $x, y, z$ are cartesian coordinates; in eqs. (4) and (5),r= $\sqrt{x^{2}+y^{2}+z^{2}}$ and $\vartheta$ is the azimuthal angle of the radius vector $r$.

Within the independent particle model, the density $\rho(\boldsymbol{r})$ for neutrons or protons ${ }^{+}$ is defined in terms of the single-particle wavefunctions $\varphi_{i}(r)$ as

$$
\rho(\boldsymbol{r})=2 \sum_{i}\left|\varphi_{i}(\boldsymbol{r})\right|^{2} v_{i}^{2}
$$

where $v_{i}^{2}$ are the usual BCS occupation probabilities. For some selected actinide nuclei at ground-state and shape-isomeric deformations, we have calculated the above moments for protons and neutrons from the wave functions. The results are presented in table 2; the moments $Q_{\lambda}$ are divided by the respective nucleon numbers in order to make the dependence on the deformation clearer. (The fission isomers are denoted by an asterisk.)

The nuclear radii at the ground states are known ${ }^{19}$ ) to be approximately proportional to $A^{\frac{1}{3}}$. By fitting the rms radii in table 2 with the formula

$$
R_{0}^{(\tau)}=\sqrt{\frac{5}{3}\left(r_{\mathrm{rms}}^{(\tau)}\right)^{2}}=r_{0}^{(\tau)} A^{\frac{1}{3}} \quad(\tau=\mathrm{n}, \mathrm{p})
$$

we find that, indeed, with the values

$$
r_{0}^{(\mathrm{p})}=1.20 \mathrm{fm}, \quad r_{0}^{(\mathrm{n})}=1.23 \mathrm{fm},
$$

the rms radii of all ground states in table 2 are reproduced within $1 \%$. The agreement of the proton radius $r_{0}^{(\mathrm{P})}=1.20 \mathrm{fm}$ with electron scattering data $\left.{ }^{19}\right)$ and the existence of a "neutron skin" which leads to a slightly larger radius $r_{0}^{(\text {n) }}$ are a consequence of the fact that we have used the droplet model predictions of Myers ${ }^{20}$ ) for the parameters of the Woods-Saxon potential.

Knowing the values (9) of $r_{0}^{(\tau)}$ which fit the rms radii eq. (5) one could expect that the reduced proton moments $Q_{2}^{\mathrm{p}} / Z$ and $Q_{4}^{\mathrm{p}} / Z$ are smaller than the corresponding neutron moments. However, the numbers in table 2 show that just the opposite is true: both reduced moments are considerably larger for protons than for the neutrons. Since the deformation of the average nuclear potential by definition is the same for protons and neutrons, this effect can only be due to the Coulomb field: it pushes the protons away from each other and enlarges the average deformation of the charge distribution. This is also reflected in the axis ratios $q_{\tau}$.

This effect of the Coulomb field has to be taken into account, if one wants to relate the theoretical ground-state deformations of the average potential directly to the measured multipole moments without going over the single-particle wave functions in

+ We will use indices $\mathrm{n}$ and $\mathrm{p}$ for neutrons and protons only where it is necessary. 
eq. (7). Since such a procedure has been used frequently in the literature, we will discuss it here in some more detail.

In fact, one can avoid the use of the wave functions in eq. (7) in calculating the multipole moments (4), if one makes use of the approximate self-consistency of the shell Inodel wave functions at equilibrium deformations, which means that the density distributions follow closely the average potential at these points ${ }^{7,18}$ ). Thus one often parametrizes the density (7) by a smooth distribution $\tilde{\rho}(\boldsymbol{r})$ (e.g. of the Fermi type) with a half-density radius $R_{0}(\vartheta)$ which for axially and left-right symmetric deformations can be defined as

$$
R(\vartheta)=R_{0}\left[1+b_{0}+\sum_{\lambda=2,4, \ldots} \beta_{\lambda} Y_{\lambda, 0}(\vartheta)\right]
$$

The constant $b_{0}$ in eq. (10) is determined as a function of $\beta_{\lambda}$ by the volume conservation condition. Assuming a constant "sharp surface" radial distribution $\tilde{\rho}(\boldsymbol{r})$, the moments $\widetilde{Q}_{2}$ and $\widetilde{Q}_{4}$ (for protons) are given by

$$
\begin{aligned}
\widetilde{Q}_{2}=\frac{3}{\sqrt{5 \pi}} Z R_{0}^{2}\left\{\beta_{2}+0.360 \beta_{2}^{2}+0.967 \beta_{2} \beta_{4}\right. & +0.328 \beta_{4}^{2} \\
& \left.+0.023 \beta_{2}^{3}-0.021 \beta_{2}^{4}+0.499 \beta_{2}^{2} \beta_{4}\right\}, \\
\widetilde{Q}_{4}=\frac{1}{\sqrt{\pi}} Z R_{0}^{4}\left\{\beta_{4}+0.725 \beta_{2}^{2}+0.983 \beta_{2} \beta_{4}+\right. & 0.411 \beta_{4}^{2} \\
& \left.+0.416 \beta_{2}^{3}+1.656 \beta_{2}^{2} \beta_{4}+0.055 \beta_{2}^{4}\right\} .
\end{aligned}
$$

(We use here the symbols $\widetilde{Q}_{i}$ to make a distinction from the actual moments $Q_{\lambda}$ obtained with the wave functions, i.e. with eqs. (7) and (4).) Eqs. (11) and (12) are exact up to terms of order $\beta_{2}^{5}, \beta_{4}^{3}, \beta_{2}^{2} \beta_{4}^{2}$, etc.

In the appendix, we derive the analogous formulae for $\widetilde{Q}_{\lambda}$ for a Fermi type distribution $\tilde{\rho}(\boldsymbol{r})$. We show there, too, that the dependence of $\widetilde{Q}_{\lambda}$ on the surface thickness $a$ of this distribution is not unique and that one therefore can use the case $a=0$, which leads to eqs. (11) and (12), without loosing accuracy.

In order to relate the parameters $\beta_{2}$ and $\beta_{4}$ (for $\beta_{6}=\beta_{8}=\ldots=0$ ) with the deformation parameters $c$ and $h$ actually used in our calculations, we used the method presented by Pauli ${ }^{12}$ ) which up to deformations of the second minimum in the actinides agrees closely with the slightly different method used by Götz et al. ${ }^{5}$ ).

Instead of using different deformations for protons and neutrons, we tried to accoum for the Coulomb effect discussed above by renormalizing the proton radius $R_{0}^{(p)}$, when using eqs. (11) and (12) for the multipole moments. For the same cases as in table 2 we calculated the moments $\widetilde{Q}_{2}$ and $\widetilde{Q}_{4}$ with the radii

$$
r_{0}^{(\mathrm{p})}=1.27 \mathrm{fm}, \quad r_{0}^{(\mathrm{n})}=1.23 \mathrm{fm},
$$

and $R_{0}^{(\tau)}=r_{0}^{(\tau)} A^{\frac{1}{3}}$. The results are shown in table 3 along with the values of $\beta_{2}$ and $\beta_{4}$ found for these cases. Comparing with the results in table 2 , we see that the quadrupole moments $\widetilde{Q}_{2}$ of the potential agree closely with the $Q_{2}$ of the actual density 
distributions both for protons and neutrons. For the case of the neutrons, this result just reflects the expected approximate self-consistency of the field, which seems to hold at least for the quadrupole moments. For the protons it means that one would underestimate the quadrupole moments by about $12 \%$ by neglecting the influence of the Coulomb field on the charge distribution.

This result is different from that of Nilsson ${ }^{21}$ ) who concluded that the quadrupole moments of the charge distributions are smaller than those of the potential, if a radius $R_{0}$ is used which approximately reproduces the rms radii. However, the difference is explained with the fact that no Coulomb potential was used in the Nilsson model of ref. ${ }^{21}$ ). We expect thus that in all calculations in which a Coulomb field is explicitly added to the average nuclear proton potential (see e.g. the recent work of Möller et al. ${ }^{22}$ )), the same effect should be found that the charge quadrupole moments are larger than those of the potential.

TABLE 3

Multipole moments calculated from the deformation of the potential (parameters $\beta_{2}, \beta_{4}$ obtained as described in the text)

\begin{tabular}{lcccccc}
\hline Nucleus & $\beta_{2}$ & $\beta_{4}$ & $\bar{Q}_{2}{ }^{n} / N$ & $\bar{Q}_{2}{ }^{p} / Z$ & $\bar{Q}_{4}{ }^{n} / N$ & $\bar{Q}_{4}^{p} / Z$ \\
\hline${ }^{230} \mathrm{Th}$ & 0.192 & 0.090 & 9.7 & 10.3 & 265 & 301 \\
${ }^{232} \mathrm{Th}$ & 0.208 & 0.087 & 10.5 & 11.2 & 275 & 312 \\
${ }^{23} \mathrm{U}$ & 0.208 & 0.087 & 10.6 & 11.3 & 278 & 316 \\
${ }^{236} \mathrm{U}$ & 0.224 & 0.078 & 11.4 & 12.2 & 274 & 311 \\
${ }^{238} \mathrm{U}$ & 0.228 & 0.066 & 11.6 & 12.3 & 249 & 284 \\
${ }^{238} \mathrm{Pu}$ & 0.229 & 0.070 & 11.7 & 12.4 & 261 & 296 \\
${ }^{240} \mathrm{Pu}$ & 0.233 & 0.061 & 11.9 & 12.6 & 244 & 278 \\
${ }^{242} \mathrm{Pu}$ & 0.235 & 0.042 & 11.8 & 12.6 & 199 & 226 \\
${ }^{244} \mathrm{Pu}$ & 0.238 & 0.033 & 11.9 & 12.7 & 179 & 204 \\
${ }^{244} \mathrm{Cm}$ & 0.238 & 0.033 & 11.9 & 12.7 & 180 & 204 \\
${ }^{246} \mathrm{Cm}$ & 0.238 & 0.033 & 12.0 & 12.8 & 181 & 206 \\
${ }^{248} \mathrm{Cm}$ & 0.248 & 0.029 & 12.5 & 13.4 & 182 & 206 \\
${ }^{232} \mathrm{Th}{ }^{23}$ & 0.604 & 0.095 & 35.1 & 37.4 & 106 & 121 \\
${ }^{236} \mathrm{U}^{*}$ & 0.630 & 0.084 & 37.1 & 39.1 & 113 & 127 \\
${ }^{236} \mathrm{Pu}^{*}$ & 0.625 & 0.078 & 36.3 & 38.7 & 108 & 122 \\
${ }^{238} \mathrm{Pu}^{*}$ & 0.637 & 0.079 & 37.4 & 39.8 & 113 & 129 \\
${ }^{240} \mathrm{Pu}^{*}$ & 0.646 & 0.082 & 38.1 & 40.7 & 119 & 135 \\
${ }^{246} \mathrm{Cm}^{*}$ & 0.670 & 0.084 & 40.8 & 43.5 & 133 & 151 \\
${ }^{254} \mathrm{Fm}^{*}$ & 0.596 & 0.061 & 35.4 & 37.8 & 101 & 115 \\
\hline
\end{tabular}

The radius constants $r_{0}(\tau)$ of eq. (I3) are used. Units as in table 2.

Strictly speaking, one should also take this Coulomb effect into account in calculating the liquid drop model (LDM) part of the deformation energy. It is however not clear to which extent the surface energy would be increased by an enlarged deformation of the protons only, and therefore the balance of the surface and Coulomb energies might shift the equilibrium deformation in either direction. We expect, though, that this shift would be small in the region of nuclei considered in this paper, since the LDM energy is quite flat here and the equilibrium shapes are mainly deter- 
mined by the shell-correction part of the total energy. In any case, such a change would affect both the equilibrium parameters $\beta_{2}$ and the charge quadrupole moments $Q_{2}$, and our conclusions drawn about their relation would essentially remain the same.

The hexadecapole moments $\widetilde{Q}_{4}$ in table 3 do not reproduce the exact values of table 2 as well as the quadrupole moments; especially the neutron moments are off in some cases by more than $40 \%$. Thus the self-consistency argument is not valid for the hexadecapole moments. For the protons, the discrepancy between $\widetilde{Q}_{4}$ and $Q_{4}$ is however not larger than $\approx 8 \%$, which, in view of the numerical error discussed below, is still a sufficient accuracy.

We should finally point out that, in addition to the above discussed errors which are inherent in the single-particle model used, some numerical errors may occur in the extraction of the equilibrium deformations due to the graphical interpolation of the potential energy surfaces ${ }^{7,12}$ ). For the values of $\beta_{2}$, these errors are not larger than $\approx 2 \%$; the $\beta_{4}$ values are, however, less accurate due to the softness of the energy surfaces in the $\beta_{4}$ direction ${ }^{12}$ ). Thus the absolute error in $\beta_{4}$ is estimated to be \pm 0.005 at the ground states and \pm 0.01 at the isomeric states. Regarding the partially small values of $\beta_{4}$, this may imply rather large relative errors in some cases.

In view of these numerical uncertainties in the values of $\beta_{2}$ and $\beta_{4}$, we can thus conclude that one may use the relations (11) and (12) together with the radii constants (13) to calculate the charge multipole moments directly from the equilibrium deformations of the potential. In detailed comparisons with the experiment, however, it might be wise to calculate the moments from the actual proton distributions, as is done in sect. 3 below.

\subsection{MOMENTS OF INERTIA}

Using the single-particle energies $\varepsilon_{i}$ and wave functions $\varphi_{i}(\boldsymbol{r})$ at a given deformation, the moments of inertia can be calculated within the cranking model ${ }^{14}$ ). In the temperature-dependent BCS formalism the moments of inertia $\mathscr{J}_{\|}$and $\mathscr{J}_{\perp}$ for rotation around the symmetry $(z-)$ axis and around an axis perpendicular to it, are given by Grin $^{23}$ ) (see also ref. ${ }^{7}$ )) as

$$
\begin{gathered}
\mathscr{J}_{\|}=\frac{\hbar^{2}}{2 T} \sum_{i} \frac{\Omega_{i}^{2}}{\cosh ^{2}\left(E_{i} / 2 T\right)}, \\
\mathscr{F}_{\perp}=\hbar^{2} \sum_{i, k}\left\{\frac{\left(u_{i} v_{k}-u_{k} v_{i}\right)^{2}}{E_{i}+E_{k}}\left[\operatorname{tgh}\left(\frac{E_{i}}{2 T}\right)+\operatorname{tgh}\left(\frac{E_{k}}{2 T}\right)\right]\right. \\
\left.+\frac{\left(u_{i} u_{k}+v_{i} v_{k}\right)^{2}}{E_{i}-E_{k}}\left[\operatorname{tgh}\left(\frac{E_{i}}{2 T}\right)-\operatorname{tgh}\left(\frac{E_{k}}{2 T}\right)\right]\right\}\left|\left\langle i\left|j_{x}\right| k\right\rangle\right|^{2} .
\end{gathered}
$$

In these equations, $E_{i}$ are the quasi-particle energies and $u_{i}, v_{i}$ the BCS occupation numbers, while $j_{x}$ is the $x$-component of the angular momentum operator and $\Omega_{i}$ the eigenvalue of its $z$-component $j_{z}$ (which commutes with the single-particle Hamilto- 

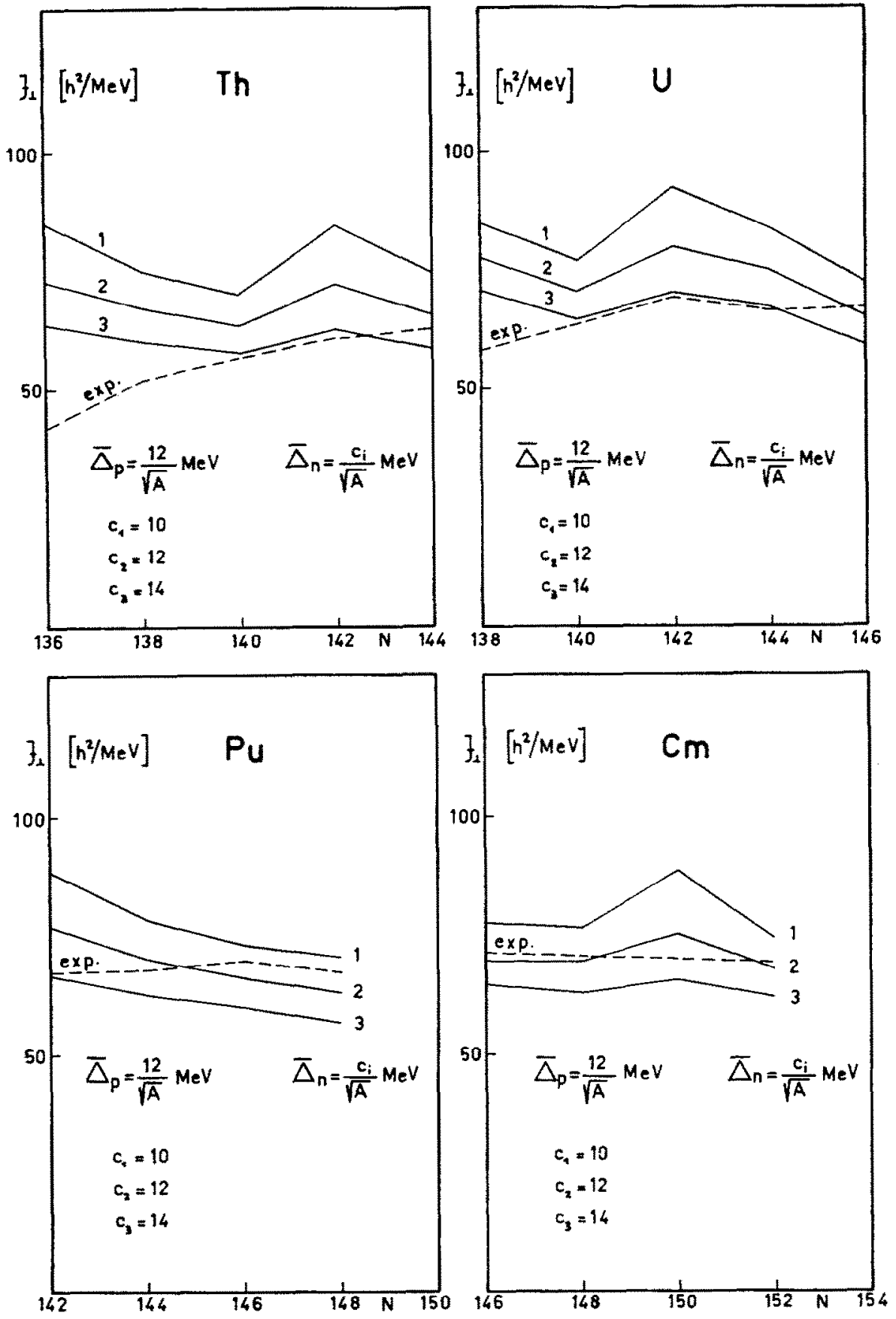

Fig. 2. Moments of inertia $\mathscr{I}_{\perp}$ at $T=0$ as functions of neutron number for different isotopes. The neutron pairing strength is varied through $c_{\mathrm{n}}$ in eq. (2). The dashed curve is drawn through experimental points. 
nian) in the $i$ th state. In the limit $T=0$, the parallel moment disappears, i.e. $\mathscr{J}_{\|}=0$. Also, the second term of the perpendicular moment $\mathscr{J}_{\perp}$ vanishes in this limit, while the first term of $\mathscr{J}_{\perp}$ reduces to the usual cranking model expression.

The pairing dependence of $\mathscr{J}_{\perp}$ at zero temperature is illustrated in fig. 2 for a series of isotopes of $\mathrm{Th}, \mathrm{U}, \mathrm{Pu}$, and $\mathrm{Cm}$. The neutron pairing strength $c_{\mathrm{n}}$ in eq. (2) is varied while $c_{\mathrm{p}}=12 \mathrm{MeV}$ is fixed. For comparison, the experimental values are shown by

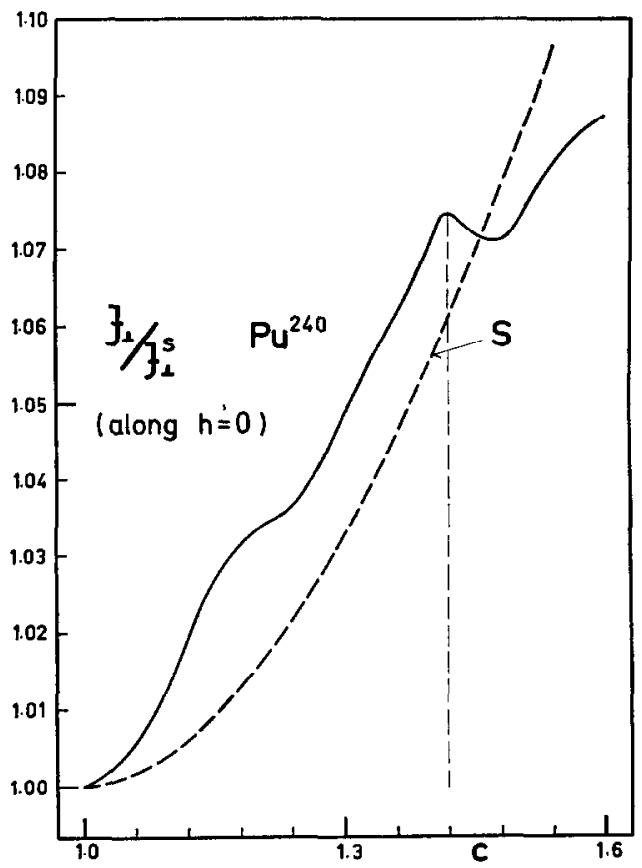

Fig. 3. The ratio $\mathscr{J}_{\perp} / \mathscr{J}_{\perp} s$ of the moments of inertia for ${ }^{240} \mathrm{Pu}$ as function of deformation. Here $\mathscr{J}_{\perp} s$ is evaluated with a pairing strength proportional to the surface, while $\mathscr{J}_{\perp}$ is obtained with a constant pairing strength. The dashed curve $S$ shows the surface area of the deformed nucleus in units of that of a sphere.

the dashed lines. For the heavier isotopes a value of $c_{\mathrm{n}}=12 \mathrm{MeV}$ fits well on the average, while a larger value is favoured for lighter isotopes. An increase of $c_{\mathrm{n}}$ and $c_{\mathrm{p}}$ (and therefore of $\Delta_{\mathrm{n}}$ and $\Delta_{\mathrm{p}}$ ) by $10 \%$ decreases the value of $\mathscr{J}_{\perp}$ by $10-15 \%$ for the nuclci considered herc. Thus the choicc of the pairing parameters is quitc crucial for the moments of inertia.

Because we want to introduce as few parameters as possible we continue with the values $c_{\mathrm{n}}=c_{\mathrm{p}}=12 \mathrm{MeV}$ previously used ${ }^{7}$ ). The disagreement with experiments for the lighter isotopes is not serious since deviations from the pure rotational model occur for the same nuclei (see sect. 3).

The deformation dependence of the pairing strength discussed above is therefore important for the moments of inertia. The size of the effect is shown in fig. 3. The 
deformation-dependent pairing strength decreases $\mathscr{J}_{\perp}$ by $\approx 3 \%$ at the ground-state deformation $(c \approx 1.2)$ and by $7-8 \%$ at a typical isomer deformation $(c \approx 1.4)$. As we shall see below, this difference is not large enough to decide on the deformation dependence of pairing by using the experimental results of $\mathscr{J}_{\perp}$.

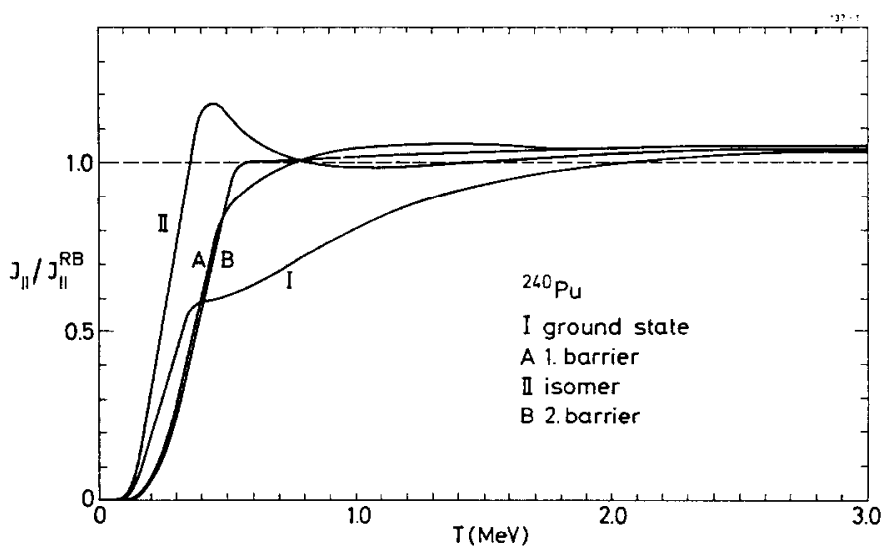

Fig. 4a. The parallel moment of inertia $\mathscr{J}_{\|}$(in rigid body units, see eq. (15a)) as function of the temperature $T$. Typical deformations of ${ }^{240} \mathrm{Pu}$ are chosen.

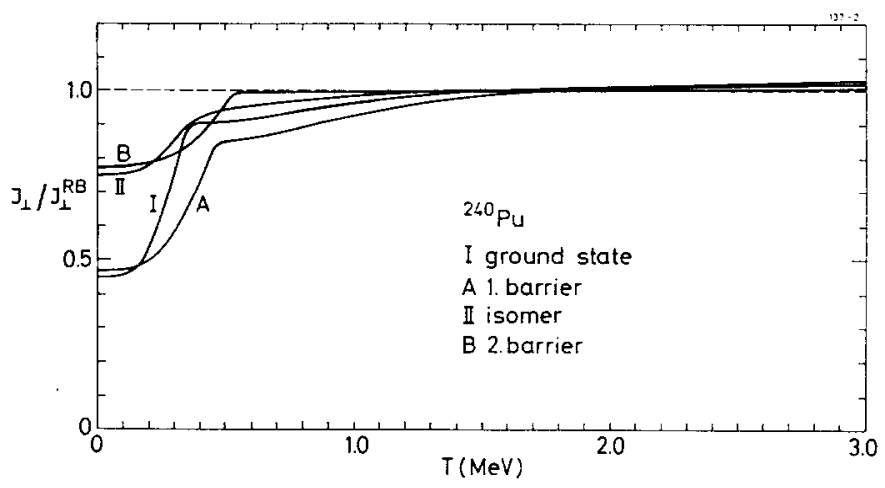

Fig. 4b. The same as fig. 4 a for the perpendicular moment of inertia, $\mathscr{J}_{\perp}$.

It has been argued ${ }^{24,26}$ ) that for a system of independent particles in a deformed well, the moments of inertia should approach their rigid body values in the limit of large nucleon numbers. The latter are defined by

$$
\begin{aligned}
& \mathscr{J}_{\|}^{\mathrm{RB}}=\int \rho(\boldsymbol{r})\left(x^{2}+y^{2}\right) \mathrm{d} \tau, \\
& \mathscr{J}_{\perp}^{\mathrm{RB}}=\int \rho(\boldsymbol{r})\left(x^{2}+z^{2}\right) \mathrm{d} \tau,
\end{aligned}
$$


where $\rho(r)$ is given by eq. (7). "Large nucleon numbers" can here be substituted by ${ }^{18}$ ) "nuclei without shell structure". Once this is realized, the behaviour of $\mathscr{J}_{\|}$and $\mathscr{J}_{\perp}$ discussed in the following can easily be understood.

For a few typical deformations of ${ }^{240} \mathrm{Pu}, \mathscr{J}_{\|} / \mathscr{J}_{\|}^{\mathrm{RB}}$ and $\mathscr{J}_{\perp} / \mathscr{J}_{\perp}^{\mathrm{RB}}$ are plotted in figs. $4 \mathrm{a}$ and $4 \mathrm{~b}$ as functions of the temperature $T$. Asymptotic values are reached for $T \gtrsim 2 \mathrm{MeV}$ when the shell effects have disappeared. In spite of the strong deformation dependence, these limits are within $2-3 \%$ equal to the rigid body values of eqs. (15). The sharp increase in the region $T \approx 0.2-0.5 \mathrm{MeV}$ is due to the disappearance of the gaps in this interval. As soon as $\Delta_{n}=\Delta_{\mathrm{p}}=0$, we have a system of independent particles still containing some shell structurc. Above the critical temperature $T \approx 0.5 \mathrm{MeV}$, the rigid body value is essentially reached, except for the small deformations (see curves I and A) for which a higher temperature is needed to wash out the shell effects $\left.{ }^{16,18}\right)$.

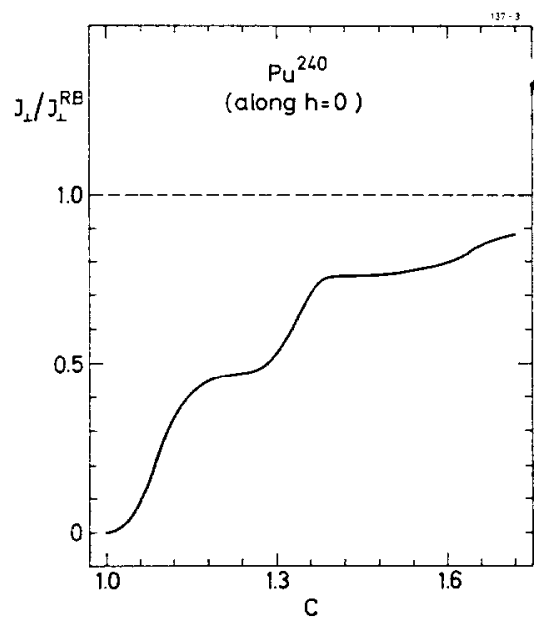

Fig. 5. The perpendicular moment of inertia (in rigid body units) as a function of deformation $c$.

The rigid body value of $\mathscr{J}_{\perp}$ is also reached at zero temperature in the limit of large deformations. This is demonstrated in fig. 5 , where $\mathscr{J}_{\perp}$ for ${ }^{240} \mathrm{Pu}$ is plotted as a function of the elongation parameter $c$. The shell structure at small deformations is clearly seen. The bumps around the deformations of the two minima $(c \approx 1.2$ and 1.4$)$ are due to the low level density leading to small pairing gaps which because of the approximate inverse proportionality in turn produce large $\mathscr{J}_{\perp}$ values. At large deformations, the rigid body value is approached although the pairing correlation still is present. This indicates that a nucleus without shell structure has a rigid body moment of inertia; the important assumption is not that the system consists of independent particles.

Since quantum mechanically a rotation around the symmetry axis is not possible, the discussion above does not hold for the parallel moment $\mathscr{J}_{\|}$at $T=0$. 
TABLE 4

Ground-state deformations and moments of inertia $\mathscr{J}_{\perp}$ (for rotation around an axis perpendicular to the symmetry axis) for nuclei with proton and mass number $Z$ and $A$

\begin{tabular}{|c|c|c|c|c|c|c|}
\hline$Z$ & $A$ & $c$ & $h$ & $\beta_{2}$ & $\beta_{4}$ & $\mathscr{F}_{\perp}\left[\frac{\hbar^{2}}{\mathrm{MeV}}\right]$ \\
\hline \multirow[t]{18}{*}{82} & 208 & 1.015 & -0.075 & -0.003 & 0.020 & 0 \\
\hline & 210 & 1.015 & -0.060 & 0.003 & 0.016 & 0 \\
\hline & 212 & 1.010 & -0.040 & 0.002 & 0.010 & 0 \\
\hline & 214 & 1.000 & 0.000 & 0.000 & 0.000 & 0 \\
\hline & 216 & 1.000 & 0.000 & 0.000 & 0.000 & 0 \\
\hline & 218 & 1.000 & 0.000 & 0.000 & 0.000 & 0 \\
\hline & 220 & 1.000 & 0.000 & 0.000 & 0.000 & 0 \\
\hline & 222 & 1.180 & -0.195 & 0.162 & 0.068 & 42 \\
\hline & 224 & 1.190 & -0.170 & 0.189 & 0.063 & 48 \\
\hline & 226 & 1.195 & -0.150 & 0.208 & 0.058 & 50 \\
\hline & 228 & 1.185 & -0.100 & 0.229 & 0.042 & 51 \\
\hline & 230 & 1.180 & -0.070 & 0.242 & 0.032 & 54 \\
\hline & 232 & 1.180 & -0.045 & 0.258 & 0.024 & 63 \\
\hline & 234 & 1.155 & 0.000 & 0.250 & 0.007 & 54 \\
\hline & 236 & 1.125 & 0.070 & 0.242 & -0.019 & 49 \\
\hline & 238 & 1.125 & 0.070 & 0.242 & -0.019 & 46 \\
\hline & 240 & 1.120 & 0.075 & 0.236 & -0.021 & 44 \\
\hline & 242 & 1.115 & 0.080 & 0.231 & -0.023 & 43 \\
\hline \multirow[t]{18}{*}{84} & 210 & 1.020 & -0.075 & 0.005 & 0.020 & 1 \\
\hline & 212 & 1.015 & -0.060 & 0.003 & 0.016 & 1 \\
\hline & 214 & 1.017 & -0.052 & 0.009 & 0.013 & 2 \\
\hline & 216 & 1.020 & -0.045 & 0.016 & 0.011 & 2 \\
\hline & 218 & 1.020 & -0.037 & 0.019 & 0.009 & 2 \\
\hline & 220 & 1.120 & -0.187 & 0.094 & 0.057 & 23 \\
\hline & 222 & 1.170 & -0.220 & 0.135 & 0.074 & 38 \\
\hline & 224 & 1.185 & -0.210 & 0.158 & 0.073 & 42 \\
\hline & 226 & 1.195 & -0.180 & 0.189 & 0.066 & 50 \\
\hline & 228 & 1.200 & -0.150 & 0.214 & 0.059 & 53 \\
\hline & 230 & 1.195 & -0.120 & 0.228 & 0.049 & 52 \\
\hline & 232 & 1.180 & -0.075 & 0.238 & 0.033 & 54 \\
\hline & 234 & 1.175 & -0.045 & 0.251 & 0.024 & 61 \\
\hline & 236 & 1.165 & -0.015 & 0.256 & 0.013 & 56 \\
\hline & 238 & 1.145 & 0.055 & 0.267 & -0.012 & 53 \\
\hline & 240 & 1.130 & 0.060 & 0.245 & -0.015 & 46 \\
\hline & 242 & 1.120 & 0.075 & 0.236 & -0.021 & 44 \\
\hline & 244 & 1.115 & 0.080 & 0.231 & -0.023 & 43 \\
\hline \multirow[t]{10}{*}{86} & 212 & 1.020 & -0.060 & 0.010 & 0.016 & 1 \\
\hline & 214 & 1.020 & -0.055 & 0.012 & 0.014 & 2 \\
\hline & 216 & 1.020 & -0.037 & 0.019 & 0.009 & 2 \\
\hline & 218 & 1.020 & -0.030 & 0.022 & 0.007 & 2 \\
\hline & 220 & 1.020 & -0.015 & 0.028 & 0.003 & 2 \\
\hline & 222 & 1.180 & -0.245 & 0.132 & 0.082 & 42 \\
\hline & 224 & 1.195 & -0.240 & 0.151 & 0.083 & 45 \\
\hline & 226 & 1.205 & -0.225 & 0.171 & 0.081 & 48 \\
\hline & 228 & 1.215 & -0.200 & 0.198 & 0.076 & 60 \\
\hline & 230 & 1.220 & -0.170 & 0.225 & 0.068 & 61 \\
\hline
\end{tabular}


TABlE 4 (continued)

\begin{tabular}{|c|c|c|c|c|c|c|}
\hline$Z$ & $A$ & $c$ & $h$ & $\beta_{2}$ & $\beta_{4}$ & $\mathscr{J}_{\perp}\left[\frac{\hbar^{2}}{\mathrm{MeV}}\right]$ \\
\hline & 232 & 1.205 & -0.135 & 0.231 & 0.055 & 56 \\
\hline & 234 & 1.185 & -0.090 & 0.235 & 0.039 & 55 \\
\hline & 236 & 1.180 & -0.090 & 0.228 & 0.038 & 60 \\
\hline & 238 & 1.170 & -0.020 & 0.260 & 0.015 & 58 \\
\hline & 240 & 1.160 & 0.008 & 0.263 & 0.005 & 54 \\
\hline & 242 & 1.135 & 0.045 & 0.245 & -0.010 & 46 \\
\hline & 244 & 1.125 & 0.070 & 0.242 & -0.019 & 45 \\
\hline & 246 & 1.120 & 0.075 & 0.236 & -0.021 & 45 \\
\hline \multirow[t]{18}{*}{88} & 214 & 1.020 & -0.060 & 0.010 & 0.016 & 0 \\
\hline & 216 & 1.020 & -0.037 & 0.019 & 0.009 & 1 \\
\hline & 218 & 1.020 & -0.030 & 0.022 & 0.007 & 2 \\
\hline & 220 & 1.025 & -0.015 & 0.036 & 0.003 & 3 \\
\hline & 222 & 1.175 & -0.245 & 0.126 & 0.081 & 44 \\
\hline & 224 & 1.200 & -0.245 & 0.153 & 0.086 & 52 \\
\hline & 226 & 1.225 & -0.255 & 0.172 & 0.093 & 59 \\
\hline & 228 & 1.225 & -0.232 & 0.188 & 0.086 & 57 \\
\hline & 230 & 1.225 & 0.210 & 0.203 & 0.080 & 65 \\
\hline & 232 & 1.220 & -0.172 & 0.223 & 0.068 & 63 \\
\hline & 234 & 1.215 & -0.150 & 0.233 & 0.061 & 61 \\
\hline & 236 & 1.185 & -0.090 & 0.235 & 0.039 & 57 \\
\hline & 238 & 1.180 & -0.070 & 0.242 & 0.032 & 64 \\
\hline & 240 & 1.175 & -0.037 & 0.256 & 0.021 & 58 \\
\hline & 242 & 1.165 & -0.008 & 0.260 & 0.011 & 55 \\
\hline & 244 & 1.150 & 0.030 & 0.260 & -0.003 & 52 \\
\hline & 246 & 1.130 & 0.060 & 0.245 & -0.015 & 47 \\
\hline & 248 & 1.120 & 0.075 & 0.236 & -0.021 & 46 \\
\hline \multirow[t]{18}{*}{90} & 216 & 1.015 & -0.045 & 0.008 & 0.012 & 0 \\
\hline & 218 & 1.015 & -0.037 & 0.011 & 0.009 & 0 \\
\hline & 220 & 1.020 & -0.022 & 0.025 & 0.005 & 1 \\
\hline & 222 & 1.020 & -0.008 & 0.030 & 0.001 & 2 \\
\hline & 224 & 1.190 & -0.245 & 0.142 & 0.084 & 51 \\
\hline & 226 & 1.230 & -0.270 & 0.167 & 0.098 & 70 \\
\hline & 228 & 1.235 & -0.250 & 0.182 & 0.095 & 66 \\
\hline & 230 & 1.235 & -0.240 & 0.192 & 0.090 & 64 \\
\hline & 232 & 1.240 & -0.225 & 0.208 & 0.087 & 73 \\
\hline & 234 & 1.225 & -0.180 & 0.224 & 0.072 & 66 \\
\hline & 236 & 1.220 & -0.157 & 0.234 & 0.064 & 62 \\
\hline & 238 & 1.195 & -0.112 & 0.234 & 0.047 & 59 \\
\hline & 240 & 1.185 & -0.075 & 0.245 & 0.034 & 66 \\
\hline & 242 & 1.180 & -0.040 & 0.262 & 0.023 & 61 \\
\hline & 244 & 1.165 & -0.015 & 0.256 & 0.013 & 55 \\
\hline & 246 & 1.150 & 0.015 & 0.251 & 0.001 & 52 \\
\hline & 248 & 1.135 & 0.040 & 0.242 & 0.008 & 48 \\
\hline & 250 & 1.125 & 0.060 & 0.237 & -0.016 & 48 \\
\hline \multirow[t]{3}{*}{92} & 218 & 1.012 & -0.042 & 0.005 & 0.011 & 0 \\
\hline & 220 & 1.015 & -0.030 & 0.014 & 0.007 & 0 \\
\hline & 222 & 1.015 & -0.030 & 0.014 & 0.007 & 0 \\
\hline
\end{tabular}


TABle 4 (continued)

\begin{tabular}{|c|c|c|c|c|c|c|}
\hline$Z$ & $A$ & $c$ & $h$ & $\beta_{2}$ & $\beta_{4}$ & $\mathscr{I}_{\perp}\left[\frac{\hbar^{2}}{\mathrm{MeV}}\right]$ \\
\hline & 224 & 1.175 & -0.240 & 0.129 & 0.080 & 42 \\
\hline & 226 & 1.215 & -0.262 & 0.157 & 0.093 & 67 \\
\hline & 228 & 1.240 & -0.270 & 0.177 & 0.100 & 79 \\
\hline & 230 & 1.240 & -0.255 & 0.187 & 0.096 & 74 \\
\hline & 232 & 1.240 & -0.240 & 0.198 & 0.091 & 72 \\
\hline & 234 & 1.240 & -0.225 & 0.208 & 0.087 & 80 \\
\hline & 236 & 1.235 & -0.195 & 0.224 & 0.078 & 75 \\
\hline & 238 & 1.220 & -0.165 & 0.228 & 0.066 & 64 \\
\hline & 240 & 1.195 & -0.112 & 0.234 & 0.047 & 61 \\
\hline & 242 & 1.180 & -0.070 & 0.242 & 0.032 & 67 \\
\hline & 244 & 1.180 & 0.055 & 0.252 & 0.027 & 61 \\
\hline & 246 & 1.175 & -0.030 & 0.261 & 0.019 & 58 \\
\hline & 248 & 1.155 & 0.008 & 0.255 & 0.004 & 53 \\
\hline & 250 & 1.135 & 0.037 & 0.240 & -0.007 & 50 \\
\hline & 252 & 1.125 & 0.055 & 0.234 & -0.014 & 49 \\
\hline \multirow[t]{18}{*}{94} & 220 & 1.010 & -0.045 & 0.000 & 0.012 & 0 \\
\hline & 222 & 1.015 & -0.032 & 0.013 & 0.008 & 0 \\
\hline & 224 & 1.015 & -0.015 & 0.019 & 0.003 & 0 \\
\hline & 226 & 1.180 & -0.228 & 0.142 & 0.077 & 44 \\
\hline & 228 & 1.220 & -0.247 & 0.172 & 0.090 & 64 \\
\hline & 230 & 1.235 & -0.255 & 0.182 & 0.095 & 74 \\
\hline & 232 & 1.240 & -0.240 & 0.198 & 0.091 & 70 \\
\hline & 234 & 1.240 & -0.228 & 0.206 & 0.088 & 69 \\
\hline & 236 & 1.240 & -0.210 & 0.219 & 0.083 & 78 \\
\hline & 238 & 1.225 & -0.173 & 0.229 & 0.070 & 70 \\
\hline & 240 & 1.215 & -0.150 & 0.233 & 0.061 & 65 \\
\hline & 242 & 1.190 & -0.100 & 0.235 & 0.042 & 63 \\
\hline & 244 & 1.180 & -0.075 & 0.238 & 0.033 & 69 \\
\hline & 246 & 1.180 & -0.060 & 0.248 & 0.029 & 63 \\
\hline & 248 & 1.175 & -0.045 & $0.25 \mathrm{I}$ & 0.024 & 57 \\
\hline & 250 & 1.155 & -0.007 & 0.245 & 0.009 & 53 \\
\hline & 252 & 1.130 & 0.045 & 0.236 & -0.011 & 49 \\
\hline & 254 & 1.125 & 0.050 & 0.231 & -0.013 & 50 \\
\hline \multirow[t]{15}{*}{96} & 222 & 1.015 & -0.050 & 0.006 & 0.013 & 0 \\
\hline & 224 & 1.015 & -0.037 & 0.011 & 0.009 & 0 \\
\hline & 226 & 1.120 & -0.172 & 0.102 & 0.053 & 26 \\
\hline & 228 & 1.180 & -0.210 & 0.153 & 0.072 & 44 \\
\hline & 230 & 1.205 & -0.220 & 0.174 & 0.079 & 59 \\
\hline & 232 & 1.230 & -0.230 & 0.194 & 0.087 & 76 \\
\hline & 234 & 1.230 & -0.220 & 0.201 & 0.084 & 73 \\
\hline & 236 & 1.225 & -0.195 & 0.213 & 0.076 & 71 \\
\hline & 238 & 1.225 & -0.180 & 0.224 & 0.072 & 77 \\
\hline & 240 & 1.220 & -0.165 & 0.228 & 0.066 & 75 \\
\hline & 242 & 1.205 & -0.127 & 0.236 & 0.053 & 70 \\
\hline & 244 & 1.180 & -0.075 & 0.238 & 0.033 & 68 \\
\hline & 246 & 1.180 & -0.075 & 0.238 & 0.033 & 75 \\
\hline & 248 & 1.180 & -0.060 & 0.248 & 0.029 & 68 \\
\hline & 250 & 1.180 & -0.060 & 0.248 & 0.029 & 63 \\
\hline
\end{tabular}


TABLE 4 (continued)

\begin{tabular}{|c|c|c|c|c|c|c|}
\hline$Z$ & $A$ & $c$ & $h$ & $\beta_{2}$ & $\beta_{4}$ & $\mathscr{J}_{\perp}\left[\frac{\hbar^{2}}{\mathrm{MeV}}\right]$ \\
\hline & 252 & 1.155 & -0.010 & 0.243 & 0.010 & 53 \\
\hline & 254 & 1.125 & 0.050 & 0.231 & -0.013 & 50 \\
\hline & 256 & 1.125 & 0.060 & 0.237 & -0.016 & 52 \\
\hline \multirow[t]{18}{*}{98} & 224 & 1.015 & -0.045 & 0.008 & 0.012 & 0 \\
\hline & 226 & 1.020 & -0.045 & 0.016 & 0.011 & 0 \\
\hline & 228 & 1.125 & -0.165 & 0.112 & 0.052 & 28 \\
\hline & 230 & 1.175 & -0.205 & 0.150 & 0.070 & 41 \\
\hline & 232 & 1.190 & -0.185 & 0.180 & 0.067 & 51 \\
\hline & 234 & 1.215 & -0.210 & 0.192 & 0.078 & 64 \\
\hline & 236 & 1.220 & -0.195 & 0.207 & 0.075 & 62 \\
\hline & 238 & 1.210 & -0.165 & 0.216 & 0.065 & 62 \\
\hline & 240 & 1.210 & -0.157 & 0.222 & 0.062 & 68 \\
\hline & 242 & 1.200 & -0.130 & 0.228 & 0.053 & 66 \\
\hline & 244 & 1.185 & -0.100 & 0.229 & 0.042 & 62 \\
\hline & 246 & 1.180 & -0.075 & 0.238 & 0.033 & 64 \\
\hline & 248 & 1.180 & -0.065 & 0.245 & 0.030 & 72 \\
\hline & 250 & 1.175 & -0.052 & 0.246 & 0.026 & 63 \\
\hline & 252 & 1.175 & -0.037 & 0.256 & 0.021 & 59 \\
\hline & 254 & 1.120 & 0.052 & 0.224 & -0.014 & 50 \\
\hline & 256 & 1.120 & 0.052 & 0.224 & -0.014 & 50 \\
\hline & 258 & 1.125 & 0.060 & 0.237 & -0.016 & 53 \\
\hline \multirow[t]{18}{*}{100} & 226 & 1.020 & -0.060 & 0.010 & 0.016 & 0 \\
\hline & 228 & 1.020 & -0.045 & 0.016 & 0.011 & 0 \\
\hline & 230 & 1.120 & -0.150 & 0.113 & 0.047 & 27 \\
\hline & 232 & 1.155 & -0.170 & 0.147 & 0.057 & 35 \\
\hline & 234 & 1.183 & -0.177 & 0.176 & 0.063 & 48 \\
\hline & 236 & 1.190 & -0.158 & 0.197 & 0.059 & 52 \\
\hline & 238 & 1.195 & -0.143 & 0.213 & 0.056 & 56 \\
\hline & 240 & 1.195 & -0.135 & 0.218 & 0.053 & 58 \\
\hline & 242 & 1.190 & -0.120 & 0.222 & 0.048 & 63 \\
\hline & 244 & 1.185 & -0.090 & 0.235 & 0.039 & 63 \\
\hline & 246 & 1.180 & -0.075 & 0.238 & 0.033 & 62 \\
\hline & 248 & 1.180 & -0.068 & 0.243 & 0.031 & 65 \\
\hline & 250 & 1.180 & -0.055 & 0.252 & 0.027 & 72 \\
\hline & 252 & 1.170 & -0.040 & 0.247 & 0.021 & 63 \\
\hline & 254 & 1.145 & 0.015 & 0.243 & 0.001 & 59 \\
\hline & 256 & 1.130 & 0.050 & 0.239 & -0.012 & 55 \\
\hline & 258 & 1.125 & 0.060 & 0.237 & -0.016 & 55 \\
\hline & 260 & 1.125 & 0.060 & 0.237 & -0.016 & 56 \\
\hline \multirow[t]{9}{*}{102} & 228 & 1.020 & -0.070 & 0.007 & 0.018 & 0 \\
\hline & 230 & 1.025 & -0.045 & 0.024 & 0.011 & 1 \\
\hline & 232 & 1.120 & -0.150 & 0.113 & 0.047 & 27 \\
\hline & 234 & 1.140 & -0.150 & 0.139 & 0.049 & 32 \\
\hline & 236 & 1.175 & -0.165 & 0.174 & 0.059 & 45 \\
\hline & 238 & 1.185 & -0.150 & 0.196 & 0.056 & 50 \\
\hline & 240 & 1.185 & -0.135 & 0.206 & 0.052 & 54 \\
\hline & 242 & 1.190 & -0.120 & 0.222 & 0.048 & 58 \\
\hline & 244 & 1.185 & -0.095 & 0.232 & 0.040 & 63 \\
\hline
\end{tabular}


TABLE 4 (continued)

\begin{tabular}{|c|c|c|c|c|c|c|}
\hline$Z$ & $A$ & $c$ & $h$ & $\beta_{2}$ & $\beta_{4}$ & $\mathscr{J}_{\perp}\left[\frac{\hbar^{2}}{\mathrm{MeV}}\right]$ \\
\hline & 246 & 1.180 & -0.075 & 0.238 & 0.033 & 63 \\
\hline & 248 & 1.180 & -0.070 & 0.242 & 0.032 & 63 \\
\hline & 250 & 1.180 & -0.060 & 0.248 & 0.029 & 66 \\
\hline & 252 & 1.175 & -0.045 & 0.251 & 0.024 & 71 \\
\hline & 254 & 1.150 & 0.008 & 0.247 & 0.004 & 61 \\
\hline & 256 & 1.125 & 0.060 & 0.237 & -0.016 & 60 \\
\hline & 258 & 1.125 & 0.070 & 0.242 & -0.019 & 58 \\
\hline & 260 & 1.125 & 0.070 & 0.242 & -0.019 & 58 \\
\hline & 262 & 1.125 & 0.075 & 0.245 & -0.021 & 59 \\
\hline \multirow[t]{18}{*}{104} & 230 & 1.020 & -0.060 & 0.010 & 0.016 & 0 \\
\hline & 232 & 1.025 & -0.040 & 0.026 & 0.010 & 1 \\
\hline & 234 & 1.112 & -0.140 & 0.108 & 0.043 & 24 \\
\hline & 236 & 1.125 & -0.110 & 0.141 & 0.036 & 29 \\
\hline & 238 & 1.145 & -0.110 & 0.169 & 0.038 & 35 \\
\hline & 240 & 1.175 & -0.120 & 0.202 & 0.046 & 46 \\
\hline & 242 & 1.185 & -0.125 & 0.212 & 0.049 & 53 \\
\hline & 244 & 1.185 & -0.105 & 0.225 & 0.043 & 55 \\
\hline & 246 & 1.180 & -0.085 & 0.232 & 0.036 & 61 \\
\hline & 248 & 1.180 & -0.075 & 0.238 & 0.033 & 62 \\
\hline & 250 & 1.180 & -0.060 & 0.248 & 0.029 & 63 \\
\hline & 252 & 1.175 & -0.050 & 0.248 & 0.025 & 64 \\
\hline & 254 & 1.145 & 0.015 & 0.243 & 0.001 & 65 \\
\hline & 256 & 1.125 & 0.065 & 0.239 & -0.018 & 64 \\
\hline & 258 & 1.120 & 0.075 & 0.236 & -0.021 & 61 \\
\hline & 260 & 1.120 & 0.075 & 0.236 & -0.021 & 58 \\
\hline & 262 & 1.120 & 0.075 & 0.236 & -0.021 & 58 \\
\hline & 264 & 1.120 & 0.075 & 0.236 & -0.021 & 59 \\
\hline \multirow[t]{18}{*}{106} & 232 & 1.020 & -0.050 & 0.014 & 0.013 & 0 \\
\hline & 234 & 1.025 & -0.030 & 0.030 & 0.007 & 2 \\
\hline & 236 & 1.040 & -0.015 & 0.060 & 0.003 & 5 \\
\hline & 238 & 1.120 & -0.035 & 0.175 & 0.013 & 30 \\
\hline & 240 & 1.125 & -0.075 & 0.160 & 0.026 & 30 \\
\hline & 242 & 1.155 & -0.100 & 0.188 & 0.037 & 37 \\
\hline & 244 & 1.185 & -0.120 & 0.215 & 0.047 & 51 \\
\hline & 246 & 1.185 & -0.100 & 0.229 & 0.042 & 54 \\
\hline & 248 & 1.180 & -0.080 & 0.235 & 0.035 & 59 \\
\hline & 250 & 1.175 & -0.060 & 0.241 & 0.028 & 60 \\
\hline & 252 & 1.170 & -0.030 & 0.253 & 0.018 & 60 \\
\hline & 254 & 1.140 & 0.030 & 0.244 & -0.005 & 60 \\
\hline & 256 & 1.120 & 0.075 & 0.236 & -0.021 & 67 \\
\hline & 258 & 1.120 & 0.075 & 0.236 & -0.021 & 66 \\
\hline & 260 & 1.120 & 0.075 & 0.236 & -0.021 & 64 \\
\hline & 262 & 1.120 & 0.075 & 0.236 & -0.021 & 61 \\
\hline & 264 & 1.120 & 0.075 & 0.236 & -0.021 & 60 \\
\hline & 266 & 1.120 & 0.075 & 0.236 & -0.021 & 61 \\
\hline
\end{tabular}

The deformation parameters $c, h$ and $\beta_{2}, \beta_{4}$ are connected as described in the text. The absolute error is \pm 0.004 in $\beta_{2}$ and \pm 0.005 in $\beta_{4}$. The moments $\mathscr{J}_{\perp}$ are in units of $h^{2} / \mathrm{MeV}$; their absolute error is $\approx 2-3$ units. 
Nevertheless, it reaches the rigid body value at large temperatures (see fig. 4a). This is explained by the fact that the time spent in non-symmetric orbitals increases with temperature, although the heating of a nucleus does not on the average destroy its symmetry.

Instead of using the wave functions in the definition of $\rho(r)$, eq. (7), one can evaluate the rigid body moments (15) again from a smooth density distribution in a similar way as we did it above for the multipole moments. Expressing the corresponding values by the quantities defined above in eqs. (5) and (11), we obtain

$$
\begin{aligned}
& \tilde{\mathscr{J}}_{\|}=\frac{2}{3} A\left\langle\widetilde{\boldsymbol{r}}^{2}\right\rangle-\frac{1}{3} \widetilde{Q}_{2}, \\
& \tilde{\mathscr{I}}_{\perp}=\frac{2}{3} \boldsymbol{A}\left\langle\widetilde{\boldsymbol{r}}^{2}\right\rangle+\frac{1}{6} \widetilde{Q}_{2},
\end{aligned}
$$

where $\left\langle\tilde{\boldsymbol{r}^{2}}\right\rangle$ is the mean square radius obtained for the smooth distribution $\tilde{\rho}(\boldsymbol{r})$, too. We found that eqs. (16) with the radii (13) reproduce the actual rigid body values (15) to within $2-3 \%$.

\section{Results and comparison with experiment}

\subsection{GROUND STATES}

Table 4 gives the deformation parameters $(c, h)$ and $\left(\beta_{2}, \beta_{4}\right)$ and moments of inertia $\mathscr{J}_{\perp}(T=0)$ for nuclei with $208 \leqq A \leqq 266$ in their ground states, thus including the transition region between lead and actinides. We note that the transition between spherical and well deformed nuclei occurs at neutron numbers $N \approx 130$. There the values of $\beta_{2}$ suddenly change from 0.0 to $0.2-0.3$ and stay around these values for the heavier nuclei. The parameter $\beta_{4}$ rises to $\approx 0.1$ around $N=136$ and falls then off rather slowly.

Only a few ground-state deformations of actinide nuclei are experimentally known ${ }^{1}$ ). Although we have concluded in subsect. 2.1 that the deformation parameters $\left(\beta_{2}, \beta_{4}\right)$ of the potential may be used to calculate the moments $Q_{2}$ and $Q_{4}$, provided that the radius $R_{0}$ in eqs. (11) and (12) is suitably chosen (eq. 13), we want to compare the experimental moments with those obtained from the wave functions (see table 2). Fig. 6a shows the quadrupole moments. The theoretical values are systematically $2-5 \%$ larger than the experimental ones. As the error in the theoretical numbers is $2-3 \%$ (see subsect. 2.1 ), the agreement is very good.

The hexadecapole moments are compared in fig. $6 \mathrm{~b}$. Here the agreement is not as striking as for the quadrupole moments. The systematic behaviour of the theoretical numbers is much smoother and falls off much slower with $A$ than the experimental values. Still, for all cases except the $\mathrm{Cm}$ isotopes the theoretical values lie within the experimental error limits, which however are quite large and increase for the heavier nuclei. Since also the theoretical relative errors in $Q_{4}$ are largest for the $\mathrm{Cm}$ isotopes due to the small values of $\beta_{4}$ (see tables 3 and 4 ), the general agreement is still satisfactory. 


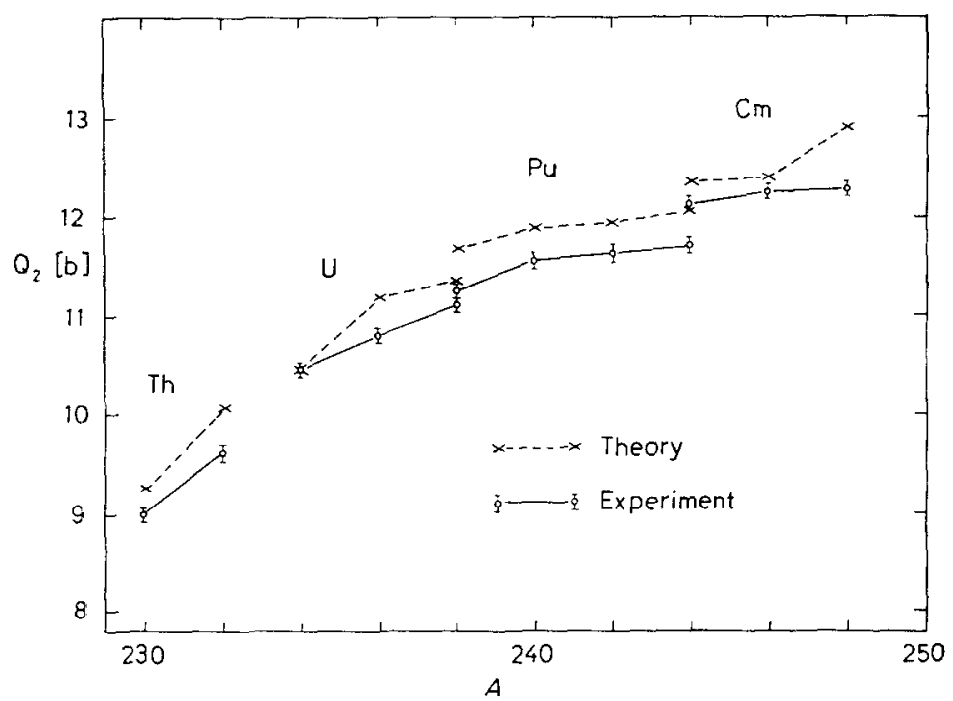

Fig. 6a. Comparison between experimental ${ }^{1}$ ) and theoretical quadrupole moments $Q_{2}$ obtained from the wave functions at the ground states.

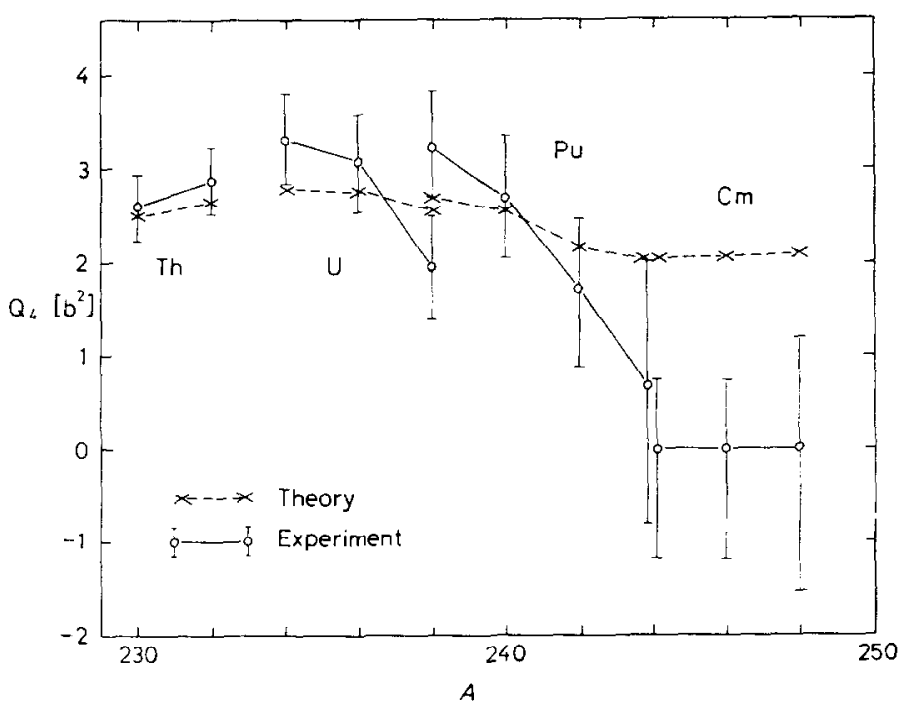

Fig. 6b. The same as fig. 6a for the hexadecapole moments, $Q_{4}$.

Very similar results have recently been obtained by Möller et al. ${ }^{22}$ ) using a deformed folded Yukawa potential. Their values of $\beta_{2}$ and $\beta_{4}$ (the latter ones for the case of the droplet model) agree with our values given in table 3 to within \pm 0.015 (absolute values). Therefore a calculation of the quadrupole moments from the wave functions 
might improve the agreement of their results with experiment (see also the discussion in subsect. 2.1).

The same quality of agreement as ours displayed in figs. $6 \mathrm{a}$ and $6 \mathrm{~b}$ is also reached in recent self-consistent calculations with a density-dependent effective nucleonnucleon interaction ${ }^{27}$ ).

TABLE 5

Comparison between experimental and theoretical moments of inertia $\mathscr{F}_{\perp}$

\begin{tabular}{|c|c|c|c|c|}
\hline Nucleus & $\begin{array}{c}a \\
\left(\mathrm{keV} / \hbar^{2}\right)\end{array}$ & $\begin{array}{c}b \\
\left(\mathrm{eV} / \hbar^{4}\right)\end{array}$ & $\begin{array}{c}\mathscr{J}_{1}^{\exp } \\
\left(\hbar^{2} / \mathrm{MeV}\right)\end{array}$ & $\mathscr{J}_{\perp}^{\text {th }} / \mathscr{J}_{\perp}{ }^{\exp }$ \\
\hline${ }^{224} \mathrm{Ra}$ & 14.7 & 101.0 & 34 & 1.53 \\
\hline${ }^{226} \mathrm{Ra}$ & 11.6 & 57.1 & 43 & 1.37 \\
\hline${ }^{228} \mathrm{Ra}$ & 10.1 & 41.7 & 50 & 1.14 \\
\hline${ }^{224} \mathrm{Th}$ & 16.1 & 107.0 & 31 & 1.64 \\
\hline${ }^{226} \mathrm{Th}$ & 12.3 & 49.8 & 41 & 1.71 \\
\hline${ }^{228} \mathrm{Th}$ & 9.7 & 18.1 & 52 & 1.27 \\
\hline${ }^{230} \mathrm{Th}$ & 9.0 & 12.4 & 56 & 1.18 \\
\hline${ }^{232} \mathrm{Th}$ & 8.4 & 10.7 & 60 & 1.23 \\
\hline${ }^{234} \mathrm{Th}$ & 8.0 & 0.0 & 63 & 1.05 \\
\hline${ }^{230} \mathrm{U}$ & 8.6 & 6.0 & 58 & 1.30 \\
\hline${ }^{232} \mathrm{U}$ & 8.0 & 7.4 & 63 & 1.14 \\
\hline${ }^{234} U$ & 7.3 & 5.4 & 69 & 1.16 \\
\hline${ }^{236} \mathrm{U}$ & 7.6 & 8.0 & 66 & 1.11 \\
\hline${ }^{238} \mathrm{U}$ & 7.5 & 3.6 & 67 & 1.00 \\
\hline${ }^{236} \mathrm{Pu}$ & 7.5 & 6.0 & 67 & 1.16 \\
\hline${ }^{238} \mathrm{Pu}$ & 7.4 & 3.7 & 68 & 1.17 \\
\hline${ }^{240} \mathrm{Pu}$ & 7.2 & 4.4 & 70 & 0.96 \\
\hline${ }^{242} \mathrm{Pu}$ & 7.4 & & 67 & 0.99 \\
\hline${ }^{244} \mathrm{Pu}$ & 7.5 & & 67 & 1.07 \\
\hline${ }^{242} \mathrm{Cm}$ & 7.1 & 6.0 & 71 & 0.99 \\
\hline${ }^{244} \mathrm{Cm}$ & 7.2 & 2.5 & 70 & 1.00 \\
\hline${ }^{246} \mathrm{Cm}$ & 7.2 & 3.6 & 70 & 1.10 \\
\hline${ }^{248} \mathrm{Cm}$ & 7.3 & 3.8 & 69 & 1.00 \\
\hline${ }^{250} \mathrm{Cf}$ & 7.0 & 0.0 & 71 & 0.89 \\
\hline${ }^{252} \mathrm{Cf}$ & 7.3 & & 68 & 0.87 \\
\hline${ }^{254} \mathrm{Fm}$ & 7.3 & 4.8 & 68 & 0.87 \\
\hline
\end{tabular}

The quantities $a$ and $b$ are defined by eq. (17). The experimental moments of inertia are given by $\mathscr{J}_{\perp}^{\text {exp }}=\hbar^{2} / 2 a$, the theoretical values $\mathscr{J}_{\perp}^{\text {th }}$ are given in table 4 . If only the $2^{+}$state is known experimentally, $a$ is obtained assuming that $b=0$ and $b$ is omitted in the table.

The moments of inertia $\mathscr{J}_{\perp}$ at the ground states can be extracted from the experimental rotational spectra. These are usually parametrized by expanding the energy in powers of its total angular momentum squared,

$$
E_{I}=a I(I+1)+b I^{2}(I+1)^{2} .
$$

In the pure rotational model, $b$ is zero and $a$ is equal to $\hbar^{2} / 2 \mathscr{J}_{\perp}$. In table 5 we list these experimental quantities together with $\mathscr{J}_{\perp}$ from table 4 for all deformed doubly even nuclei whose ground-state rotational bands have been measured ${ }^{28}$ ). In fig. 7 
the ratios between calculated and experimental moments are plotted. The results of two recent publications ${ }^{29,30}$ ) utilizing the modified harmonic oscillator are also shown for comparison.

Our results deviate most strongly from the experiment in the $\mathrm{Ra}$ and the lighter $\mathrm{Th}$ isotopes. We note from table 5, however, that there is a clear correlation between these discrepancies and the $b$-values of the same nuclei. For those nuclei with $A<238, b$ is larger than $40 \mathrm{eV} / \hbar^{4}$. This indicates that the rotational model and therefore the cranking model begin to be inappropriate for these nuclei. From table 4 we see also, that they are less deformed than the typical rotational actinides. For the

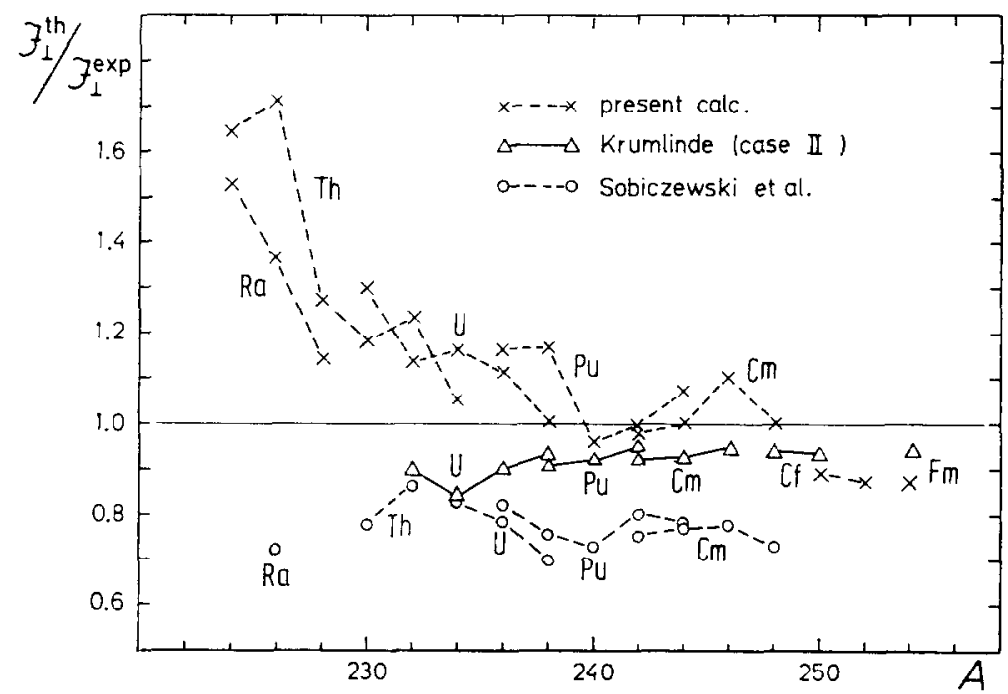

Fig. 7. Comparison between experimental and theoretical moments of inertia. The crosses are our results, circles show the calculations of ref. ${ }^{30}$ ) and triangles those of ref. ${ }^{29}$ ); in all theoretical calculations, the assumption of a constant pairing strength has been made.

rest of the nuclei, the agreement is quite satisfactory; in all cases where $b$ is less than $10 \mathrm{eV} / h^{4}$, the discrepancy between our results and the experiment is less than $20 \%$.

On the average, our results are 5-10\% too large. This is in contrast to the results by Sobiczewski et al. ${ }^{30}$ ), which on the average are $15-20 \%$ too small. With the same single-particle potential but a somewhat different treatment of the BCS pairing, Krumlinde ${ }^{29}$ ) obtained results $5-10 \%$ too low.

The main differences in the theoretical predictions come from the choice of the pairing strength. As we have discussed in subsect. 2.2, the strong dependence of $\mathscr{J}_{\perp}$ on the pairing gaps would make it easy to fit the pairing parameters to obtain a better agreement. Instead, we tried to see how well we can reproduce the experiments with only one parameter. Considering this, our results are quite satisfying. In fact, the simplified treatment of the pairing effects probably makes it unreasonable to do more detailed fits of the pairing parameters. A recent investigation ${ }^{31}$ ) shows that the 
TABLE 6

Isomeric-state deformations and moments of inertia, as in table 4

\begin{tabular}{|c|c|c|c|c|c|c|}
\hline$Z$ & $A$ & $c$ & $h$ & $\beta_{2}$ & $\beta_{4}$ & $\mathscr{J}_{\perp}\left[\frac{\hbar^{2}}{\mathrm{MeV}}\right]$ \\
\hline \multirow[t]{13}{*}{82} & 210 & 1.250 & 0.075 & 0.461 & 0.001 & 84 \\
\hline & 212 & 1.255 & 0.075 & 0.470 & 0.002 & 86 \\
\hline & 214 & 1.245 & 0.089 & 0.464 & -0.006 & 85 \\
\hline & 216 & 1.255 & 0.075 & 0.470 & 0.002 & 90 \\
\hline & 218 & 1.260 & 0.080 & 0.483 & 0.001 & 93 \\
\hline & 220 & 1.265 & 0.075 & 0.487 & 0.004 & 94 \\
\hline & 222 & 1.265 & 0.075 & 0.487 & 0.004 & 93 \\
\hline & 224 & 1.355 & 0.007 & 0.571 & 0.056 & 130 \\
\hline & 226 & 1.365 & 0.000 & 0.580 & 0.061 & 135 \\
\hline & 228 & 1.385 & -0.019 & 0.589 & 0.074 & 134 \\
\hline & 230 & 1.370 & -0.004 & 0.583 & 0.064 & 126 \\
\hline & 232 & 1.370 & 0.000 & 0.588 & 0.063 & 122 \\
\hline & 234 & 1.345 & 0.018 & 0.567 & 0.049 & 115 \\
\hline \multirow[t]{15}{*}{84} & 210 & 1.260 & 0.058 & 0.464 & 0.009 & 81 \\
\hline & 212 & 1.245 & 0.083 & 0.459 & -0.004 & 84 \\
\hline & 214 & 1.250 & 0.075 & 0.461 & 0.001 & 85 \\
\hline & 216 & 1.255 & 0.089 & 0.482 & -0.003 & 88 \\
\hline & 218 & 1.255 & 0.085 & 0.478 & -0.002 & 91 \\
\hline & 220 & 1.245 & 0.105 & 0.477 & -0.011 & 93 \\
\hline & 222 & 1.245 & 0.112 & 0.483 & -0.014 & 92 \\
\hline & 224 & 1.270 & 0.080 & 0.501 & 0.004 & 95 \\
\hline & 226 & 1.395 & -0.036 & 0.584 & 0.082 & 138 \\
\hline & 228 & 1.395 & -0.036 & 0.584 & 0.082 & 138 \\
\hline & 230 & 1.370 & 0.000 & 0.588 & 0.063 & 135 \\
\hline & 232 & 1.385 & -0.018 & 0.590 & 0.074 & 135 \\
\hline & 234 & 1.385 & -0.015 & 0.594 & 0.073 & 131 \\
\hline & 236 & 1.375 & -0.012 & 0.582 & 0.068 & 126 \\
\hline & 238 & 1.370 & 0.000 & 0.588 & 0.063 & 125 \\
\hline \multirow[t]{15}{*}{86} & 212 & 1.255 & 0.060 & 0.457 & 0.007 & 80 \\
\hline & 214 & 1.250 & 0.083 & 0.468 & -0.002 & 85 \\
\hline & 216 & 1.250 & 0.090 & 0.474 & -0.005 & 86 \\
\hline & 218 & 1.240 & 0.113 & 0.475 & -0.016 & 87 \\
\hline & 220 & 1.240 & 0.125 & 0.485 & -0.020 & 91 \\
\hline & 222 & 1.245 & 0.105 & 0.477 & -0.011 & 92 \\
\hline & 224 & 1.245 & 0.115 & 0.486 & -0.015 & 93 \\
\hline & 226 & 1.240 & 0.120 & 0.481 & -0.018 & 88 \\
\hline & 228 & 1.420 & -0.068 & 0.580 & 0.098 & 164 \\
\hline & 230 & 1.425 & -0.056 & 0.603 & 0.098 & 157 \\
\hline & 232 & 1.435 & -0.068 & 0.602 & 0.104 & 155 \\
\hline & 234 & 1.445 & -0.075 & 0.606 & 0.110 & 154 \\
\hline & 236 & 1.435 & -0.061 & 0.611 & 0.103 & 150 \\
\hline & 238 & 1.425 & -0.050 & 0.611 & 0.097 & 147 \\
\hline & 240 & 1.405 & -0.036 & 0.599 & 0.086 & 136 \\
\hline \multirow[t]{3}{*}{88} & 214 & 1.255 & 0.064 & 0.460 & 0.006 & 80 \\
\hline & 216 & 1.245 & 0.082 & 0.458 & -0.003 & 83 \\
\hline & 218 & 1.245 & 0.093 & 0.467 & -0.007 & 84 \\
\hline
\end{tabular}


MOMENTS OF INERTIA

TABLE 6 (continued)

\begin{tabular}{|c|c|c|c|c|c|c|}
\hline$Z$ & $A$ & $c$ & $h$ & $\beta_{2}$ & $\beta_{4}$ & $\mathscr{J}_{\perp}\left[\frac{\hbar^{2}}{\mathrm{MeV}}\right]$ \\
\hline & 220 & 1.240 & 0.121 & 0.481 & -0.019 & 87 \\
\hline & 222 & 1.240 & 0.128 & 0.487 & -0.021 & 90 \\
\hline & 224 & 1.245 & 0.128 & 0.497 & -0.020 & 97 \\
\hline & 226 & 1.230 & 0.140 & 0.478 & -0.028 & 90 \\
\hline & 228 & 1.280 & 0.100 & 0.537 & 0.000 & 99 \\
\hline & 230 & 1.425 & -0.066 & 0.590 & 0.100 & 157 \\
\hline & 232 & 1.425 & -0.054 & 0.606 & 0.098 & 149 \\
\hline & 234 & 1.440 & -0.071 & 0.605 & 0.107 & 148 \\
\hline & 236 & 1.455 & -0.075 & 0.621 & 0.114 & 157 \\
\hline & 238 & 1.460 & -0.075 & 0.628 & 0.116 & 157 \\
\hline & 240 & 1.425 & -0.052 & 0.608 & 0.097 & 140 \\
\hline & 242 & 1.415 & -0.032 & 0.620 & 0.089 & 138 \\
\hline \multirow[t]{15}{*}{90} & 216 & 1.250 & 0.075 & 0.461 & 0.001 & 79 \\
\hline & 218 & 1.245 & 0.095 & 0.469 & -0.008 & 83 \\
\hline & 220 & 1.245 & 0.104 & 0.476 & -0.011 & 85 \\
\hline & 222 & 1.235 & 0.136 & 0.484 & -0.025 & 88 \\
\hline & 224 & 1.235 & 0.143 & 0.490 & -0.028 & 92 \\
\hline & 226 & 1.235 & 0.150 & 0.496 & -0.031 & 98 \\
\hline & 228 & 1.230 & 0.150 & 0.486 & -0.032 & 92 \\
\hline & 230 & 1.220 & 0.164 & 0.478 & -0.039 & 86 \\
\hline & 232 & 1.420 & -0.050 & 0.604 & 0.095 & 154 \\
\hline & 234 & 1.420 & -0.046 & 0.609 & 0.094 & 147 \\
\hline & 236 & 1.430 & -0.052 & 0.616 & 0.099 & 145 \\
\hline & 238 & 1.435 & -0.052 & 0.623 & 0.102 & 143 \\
\hline & 240 & 1.420 & -0.030 & 0.630 & 0.091 & 141 \\
\hline & 242 & 1.415 & -0.012 & 0.646 & 0.085 & 140 \\
\hline & 244 & 1.410 & -0.013 & 0.636 & 0.083 & 138 \\
\hline \multirow[t]{15}{*}{92} & 218 & 1.245 & 0.081 & 0.457 & -0.003 & 80 \\
\hline & 220 & 1.245 & 0.100 & 0.473 & -0.010 & 86 \\
\hline & 222 & 1.245 & 0.114 & 0.485 & -0.015 & 88 \\
\hline & 224 & 1.240 & 0.134 & 0.492 & -0.024 & 92 \\
\hline & 226 & 1.240 & 0.142 & 0.499 & -0.027 & 96 \\
\hline & 228 & 1.255 & 0.134 & 0.521 & -0.020 & 104 \\
\hline & 230 & 1.240 & 0.150 & 0.505 & -0.030 & 98 \\
\hline & 232 & 1.400 & -0.014 & 0.619 & 0.079 & 138 \\
\hline & 234 & 1.410 & -0.030 & 0.614 & 0.087 & 143 \\
\hline & 236 & 1.410 & -0.018 & 0.630 & 0.084 & 144 \\
\hline & 238 & 1.415 & -0.012 & 0.646 & 0.085 & 150 \\
\hline & 240 & 1.410 & -0.011 & 0.639 & 0.082 & 145 \\
\hline & 242 & 1.415 & -0.010 & 0.648 & 0.084 & 143 \\
\hline & 244 & 1.410 & -0.006 & 0.646 & 0.081 & 140 \\
\hline & 246 & 1.385 & 0.034 & 0.654 & 0.060 & 135 \\
\hline \multirow[t]{6}{*}{94} & 220 & 1.255 & 0.089 & 0.482 & -0.003 & 87 \\
\hline & 222 & 1.250 & 0.107 & 0.488 & -0.011 & 92 \\
\hline & 224 & 1.250 & 0.116 & 0.496 & -0.014 & 93 \\
\hline & 226 & 1.245 & 0.134 & 0.502 & -0.022 & 97 \\
\hline & 228 & 1.245 & 0.141 & 0.508 & -0.025 & 101 \\
\hline & 230 & 1.260 & 0.121 & 0.519 & -0.014 & 107 \\
\hline
\end{tabular}


TABLE 6 (continued)

\begin{tabular}{|c|c|c|c|c|c|c|}
\hline$Z$ & $A$ & $c$ & $h$ & $\beta_{2}$ & $\beta_{4}$ & $\mathscr{J}_{\perp}\left[\frac{\hbar^{2}}{\mathrm{MeV}}\right]$ \\
\hline & 232 & 1.305 & 0.085 & 0.568 & 0.013 & 112 \\
\hline & 234 & 1.405 & -0.007 & 0.636 & 0.079 & 141 \\
\hline & 236 & 1.400 & -0.009 & 0.625 & 0.078 & 142 \\
\hline & 238 & 1.405 & -0.006 & 0.637 & 0.079 & 145 \\
\hline & 240 & 1.410 & -0.009 & 0.646 & 0.082 & 150 \\
\hline & 242 & 1.410 & -0.004 & 0.648 & 0.081 & 147 \\
\hline & 244 & 1.415 & 0.000 & 0.662 & 0.082 & 145 \\
\hline & 246 & 1.390 & 0.030 & 0.658 & 0.063 & 139 \\
\hline & 248 & 1.365 & 0.069 & 0.661 & 0.040 & 137 \\
\hline & 250 & 1.365 & 0.075 & 0.668 & 0.038 & 141 \\
\hline & 252 & 1.365 & 0.093 & 0.689 & 0.032 & 149 \\
\hline & 254 & 1.360 & 0.112 & 0.702 & 0.023 & 153 \\
\hline \multirow[t]{18}{*}{96} & 222 & 1.255 & 0.093 & 0.485 & -0.005 & 91 \\
\hline & 224 & 1.265 & 0.096 & 0.506 & -0.003 & 99 \\
\hline & 226 & 1.260 & 0.100 & 0.500 & -0.006 & 99 \\
\hline & 228 & 1.255 & 0.129 & 0.516 & -0.018 & 103 \\
\hline & 230 & 1.275 & 0.112 & 0.539 & -0.006 & 115 \\
\hline & 232 & 1.285 & 0.118 & 0.563 & -0.006 & 118 \\
\hline & 234 & 1.385 & 0.007 & 0.621 & 0.067 & 137 \\
\hline & 236 & 1.405 & 0.000 & 0.645 & 0.077 & 144 \\
\hline & 238 & 1.410 & 0.008 & 0.664 & 0.077 & 146 \\
\hline & 240 & 1.405 & -0.009 & 0.633 & 0.080 & 147 \\
\hline & 242 & 1.410 & -0.007 & 0.644 & 0.081 & 152 \\
\hline & 244 & 1.415 & 0.000 & 0.662 & 0.082 & 151 \\
\hline & 246 & 1.420 & 0.000 & 0.670 & 0.084 & 149 \\
\hline & 248 & 1.385 & 0.041 & 0.663 & 0.057 & 142 \\
\hline & 250 & 1.365 & 0.075 & 0.668 & 0.038 & 142 \\
\hline & 252 & 1.370 & 0.093 & 0.699 & 0.034 & 151 \\
\hline & 254 & 1.360 & 0.111 & 0.701 & 0.024 & 155 \\
\hline & 256 & 1.360 & 0.120 & 0.712 & 0.020 & 160 \\
\hline \multirow[t]{17}{*}{98} & 224 & 1.300 & 0.054 & 0.529 & 0.022 & 98 \\
\hline & 226 & 1.295 & 0.052 & 0.518 & 0.021 & 99 \\
\hline & 228 & 1.275 & 0.098 & 0.526 & -0.001 & 104 \\
\hline & 230 & 1.290 & 0.106 & 0.561 & 0.000 & 115 \\
\hline & 232 & 1.295 & 0.098 & 0.563 & 0.005 & 123 \\
\hline & 234 & 1.330 & 0.075 & 0.603 & 0.025 & 126 \\
\hline & 236 & 1.390 & 0.019 & 0.644 & 0.066 & 142 \\
\hline & 238 & 1.390 & 0.014 & 0.638 & 0.067 & 144 \\
\hline & 240 & 1.415 & 0.000 & 0.662 & 0.082 & 148 \\
\hline & 242 & 1.410 & 0.000 & 0.653 & 0.080 & 150 \\
\hline & 244 & 1.410 & 0.006 & 0.661 & 0.078 & 156 \\
\hline & 246 & 1.410 & 0.007 & 0.663 & 0.078 & 152 \\
\hline & 248 & 1.400 & 0.036 & 0.683 & 0.065 & 150 \\
\hline & 250 & 1.370 & 0.075 & 0.677 & 0.040 & 146 \\
\hline & 252 & 1.365 & 0.086 & 0.681 & 0.034 & 149 \\
\hline & 254 & 1.365 & 0.096 & 0.693 & 0.031 & 154 \\
\hline & 256 & 1.365 & 0.111 & 0.711 & 0.026 & 162 \\
\hline 100 & 226 & 1.335 & 0.036 & 0.570 & 0.040 & 108 \\
\hline
\end{tabular}


TABLE 6 (continued)

\begin{tabular}{|c|c|c|c|c|c|c|}
\hline$Z$ & $A$ & $c$ & $h$ & $\beta_{2}$ & $\beta_{4}$ & $\mathscr{J}_{\perp}\left[\frac{\hbar^{2}}{\mathrm{MeV}}\right]$ \\
\hline & 228 & 1.330 & 0.052 & 0.579 & 0.033 & 111 \\
\hline & 230 & 1.320 & 0.068 & 0.578 & 0.024 & 114 \\
\hline & 232 & 1.300 & 0.089 & 0.563 & 0.010 & 117 \\
\hline & 234 & 1.330 & 0.075 & 0.603 & 0.025 & 130 \\
\hline & 236 & 1.370 & 0.044 & 0.640 & 0.050 & 137 \\
\hline & 238 & 1.390 & 0.025 & 0.652 & 0.064 & 145 \\
\hline & 240 & 1.415 & 0.000 & 0.662 & 0.082 & 151 \\
\hline & 242 & 1.415 & 0.000 & 0.662 & 0.082 & 151 \\
\hline & 244 & 1.415 & 0.000 & 0.662 & 0.082 & 154 \\
\hline & 246 & 1.420 & 0.006 & 0.678 & 0.082 & 162 \\
\hline & 248 & 1.415 & 0.014 & 0.680 & 0.078 & 158 \\
\hline & 250 & 1.420 & 0.009 & 0.682 & 0.082 & 155 \\
\hline & 252 & 1.380 & 0.038 & 0.650 & 0.056 & 146 \\
\hline & 254 & 1.370 & 0.007 & 0.596 & 0.061 & 138 \\
\hline & 256 & 1.370 & 0.050 & 0.647 & 0.048 & 150 \\
\hline & 258 & 1.360 & 0.083 & 0.668 & 0.034 & 157 \\
\hline & 260 & 1.370 & 0.075 & 0.677 & 0.040 & 160 \\
\hline \multirow[t]{18}{*}{102} & 228 & 1.350 & 0.037 & 0.597 & 0.045 & 115 \\
\hline & 230 & 1.350 & 0.045 & 0.606 & 0.042 & 118 \\
\hline & 232 & 1.345 & 0.057 & 0611 & 0.036 & 120 \\
\hline & 234 & 1.350 & 0.060 & 0.623 & 0.037 & 125 \\
\hline & 236 & 1.385 & 0.042 & 0.664 & 0.057 & 138 \\
\hline & 238 & 1.400 & 0.033 & 0.679 & 0.066 & 147 \\
\hline & 240 & 1.405 & 0.027 & 0.680 & 0.070 & 154 \\
\hline & 242 & 1.405 & 0.025 & 0.678 & 0.071 & 155 \\
\hline & 244 & 1.405 & 0.025 & 0.678 & 0.071 & 155 \\
\hline & 246 & 1.410 & 0.025 & 0.686 & 0.073 & 160 \\
\hline & 248 & 1.412 & 0.025 & 0.690 & 0.074 & 166 \\
\hline & 250 & 1.415 & 0.025 & 0.695 & 0.075 & 164 \\
\hline & 252 & 1.415 & 0.033 & 0.706 & 0.073 & 162 \\
\hline & 254 & 1.400 & 0.075 & 0.734 & 0.053 & 162 \\
\hline & 256 & 1.385 & 0.080 & 0.712 & 0.045 & 160 \\
\hline & 258 & 1.385 & 0.086 & 0.719 & 0.043 & 165 \\
\hline & 260 & 1.375 & 0.106 & 0.725 & 0.032 & 170 \\
\hline & 262 & 1.365 & 0.138 & 0.744 & 0.016 & 175 \\
\hline \multirow[t]{14}{*}{104} & 238 & 1.415 & 0.037 & 0.711 & 0.072 & 156 \\
\hline & 240 & 1.415 & 0.030 & 0.702 & 0.074 & 159 \\
\hline & 242 & 1.415 & 0.025 & 0.695 & 0.075 & 165 \\
\hline & 244 & 1.415 & 0.022 & 0.691 & 0.076 & 166 \\
\hline & 246 & 1.415 & 0.022 & 0.691 & 0.076 & 165 \\
\hline & 248 & 1.415 & 0.020 & 0.688 & 0.077 & 168 \\
\hline & 250 & 1.420 & 0.020 & 0.697 & 0.079 & 176 \\
\hline & 252 & 1.420 & 0.020 & 0.697 & 0.079 & 173 \\
\hline & 254 & 1.420 & 0.022 & 0.700 & 0.078 & 170 \\
\hline & 256 & 1.405 & 0.050 & 0.710 & 0.064 & 167 \\
\hline & 258 & 1.400 & 0.064 & 0.720 & 0.057 & 169 \\
\hline & 260 & 1.400 & 0.070 & 0.727 & 0.055 & 172 \\
\hline & 262 & 1.390 & 0.093 & 0.738 & 0.043 & 177 \\
\hline & 264 & 1.370 & 0.129 & 0.743 & 0.021 & 186 \\
\hline
\end{tabular}


TABLE 6 (continued)

\begin{tabular}{|c|c|c|c|c|c|c|c|}
\hline$Z$ & $A$ & $c$ & $h$ & $\beta_{2}$ & $\beta_{4}$ & $\mathscr{J}_{\perp}$ & {$\left[\frac{\hbar^{2}}{\mathrm{MeV}}\right]$} \\
\hline \multirow[t]{13}{*}{106} & 242 & 1.455 & 0.008 & 0.741 & 0.099 & & 171 \\
\hline & 244 & 1.430 & 0.025 & 0.722 & 0.082 & & 175 \\
\hline & 246 & 1.420 & 0.020 & 0.697 & 0.079 & & 172 \\
\hline & 248 & 1.420 & 0.020 & 0.697 & 0.079 & & 171 \\
\hline & 250 & 1.420 & 0.022 & 0.700 & 0.078 & & 175 \\
\hline & 252 & 1.420 & 0.030 & 0.711 & 0.076 & & 182 \\
\hline & 254 & 1.420 & 0.030 & 0.711 & 0.076 & & 180 \\
\hline & 256 & 1.425 & 0.033 & 0.724 & 0.078 & & 178 \\
\hline & 258 & 1.425 & 0.037 & 0.729 & 0.077 & & 177 \\
\hline & 260 & 1.425 & 0.052 & 0.750 & 0.072 & & 176 \\
\hline & 262 & 1.425 & 0.055 & 0754 & 0.071 & & 179 \\
\hline & 264 & 1.420 & 0.060 & 0.752 & 0.068 & & 180 \\
\hline & 266 & 1.410 & 0.080 & 0.760 & 0.057 & & 182 \\
\hline
\end{tabular}

Absolute errors \pm 0.004 in $\beta_{2}$ and \pm 0.01 in $\beta_{4}$ and $\approx 3-6$ units in $\mathscr{F}_{\perp}$.

TABLE 7

Calculated moments for the isomeric states

\begin{tabular}{lllll}
\hline Nucleus & $Q_{2}{ }^{\mathrm{p}}$ & $Q_{4}{ }^{\mathrm{p}}$ & $\mathscr{J}_{\perp}{ }^{\text {th }}$ & $\mathscr{J}_{\perp}^{\text {exp }}$ \\
\hline${ }^{232} \mathrm{Th}^{*}$ & 33.9 & 11.8 & 153 & 141 \\
${ }^{236} \mathrm{U}^{*}$ & 36.1 & 12.3 & 144 & \\
${ }^{236} \mathrm{Pu}^{*}$ & 36.7 & 12.0 & 152 & 150 \\
${ }^{238} \mathrm{Pu}^{*}$ & 37.6 & 12.5 & 146 & \\
${ }^{240} \mathrm{Pu}^{*}$ & 38.2 & 13.0 & 147 & 152 \\
${ }^{246} \mathrm{Cm}^{*}$ & 41.5 & 14.7 & 143 & \\
${ }^{25} \mathrm{Fm}^{*}$ & 38.7 & 12.0 & & \\
\hline
\end{tabular}

Quadrupole moments $Q_{2}$ in $\mathrm{b}$, hexadecapole moments $Q_{4}$ in $\mathrm{b}^{2}$ and moments of inertia in $\hbar^{2} / \mathrm{MeV}$. The two experimental moments of inertia ${ }^{2,3}$ ) are also given.

quadrupole components of the pairing interaction may not be neglected in calculations of moments of inertia. Also the effect of the rotation on the pairing interaction should be taken into account. This gives rise to a correction to the cranking model expression (14b), the so-called Migdal term. It is shown ${ }^{31}$ ) that this term might be comparable to the discrepancies between experiment and the simple cranking model results.

\subsection{ISOMERIC STATES}

For the second minima we give in table 6 the same quantities as in table 4 . Very little experimental information is available so far. But recently, rotational bands built on the lowest $0^{+}$state were observed for ${ }^{240} \mathrm{Pu}$ [ref. ${ }^{2}$ )] and ${ }^{236} \mathrm{U}$ [ref. ${ }^{3}$ )]. Since these nuclei are almost ideal rotators in the highly deformed isomeric states, the moments of inertia can be determined and used as an indirect measurement of the 
deformation of the fission isomers. The two cases, among others, are given in table 7 . As we see, the theoretical values are within $2 \%$ of the experimental ones. The Nilsson model calculations of Sobiczewski et al. ${ }^{30}$ ) predict values of $\mathscr{J}_{\perp} 11 \%$ too large for ${ }^{236} \mathrm{U}$ and within $1 \%$ for ${ }^{240} \mathrm{Pu}$.

The surface-dependent pairing strength (see sect. 2) leads to $7-8 \%(9-11 \%$ in ref. ${ }^{30}$ )) smaller values of $\mathscr{J}_{\perp}$ for the fission isomers. Since our ground-state values are systematically too high, a surface independent strength seems here to give a better overall agreement with the experiment. However, the uncertainties and simplifications involved in the present treatment of the pairing make a final decision impossible at the present stage.

The quadrupole moments for the second minimum have not been measured directly yet. Serious attempts ${ }^{4}$ ) are being made, however. For the nuclei close to the most likely candidates for these experiments we calculated $Q_{2}$ and $Q_{4}$ from the wave functions at the second minimum. The results are given in table 7. Typically, as for ${ }^{240} \mathrm{Pu}$ and ${ }^{236} \mathrm{U}, Q_{2}$ is $3-4$ times as big as the corresponding ground-state value, i.e. $35-40 \mathrm{~b}$.

\section{Summary}

Strutinsky's shell-correction method is used to obtain the equilibrium points of the energy surface for all actinide nuclei. The average single-particle potential applied is a deformed Woods-Saxon well of constant skin thickness; the same potential parameters and pairing strength have been used as in earlier calculations ${ }^{5,7}$ ).

We study the relation between the multipole moments obtained either from the wave functions or from the deformation of the average potential. We see that care should be taken in relating the parameters $\beta_{2}, \beta_{4}$ to the moments $Q_{2}, Q_{4}$ of the charge distribution, because the Coulomb field appears to enlarge the deformation.

Moments of inertia are evaluated within the cranking model. Their dependence on temperature and deformation is investigated. In particular it is shown that the rigid body value is approached in the limit of both large temperatures and large (elongational) deformations.

Extensive tables of deformation parameters $\beta_{2}$ and $\beta_{4}$ and of moments of inertia $\mathscr{I}_{\perp}$ are given at ground states and isomeric states of actinide nuclei. Comparison with available experimental data is made. The agreement is very good, considering the fact that no parameter has been changed to improve the results. For some typical nuclei, we predict the values of $Q_{2}$ and $Q_{4}$ also at the second minimum, in the hope that the $Q_{2}$ values soon will be measured.

The authors are indebted to Prof. K. Alder and Drs. U. Götz, I. Hamamoto and Ph. Quentin for many discussions. Assistance of R. Häring with the numerical work is appreciated. Part of this work was done at the Niels Bohr Institute (M.B.), at the Weizmann Institute (T.L.) and at Nordita (A.S.J.). The hospitality and the financial support at these institutes is gratefully acknowledged. 


\section{Appendix}

MULTIPOLE MOMENTS OF A FERMI DISTRIBUTION WITH FINITE SURFACE THICKNESS

We consider an axially symmetric Fermi-type density distribution

$$
\rho(\boldsymbol{r})=\frac{\rho_{0}}{1+\exp [(r-R(\vartheta)) / a]},
$$

where the half-density radius $R(\vartheta)$ is given by

$$
R(\vartheta)=R_{0}\left\{1+b_{0}+\beta_{2} Y_{2}(\vartheta)+\beta_{4} Y_{4}(\vartheta)\right\} .
$$

The radius $R_{0}$ is constant and $b_{0}$ is a function of $\beta_{2}$ and $\beta_{4}$ determined by the volume conservation condition (see below).

Before calculating the multipole moments of the distribution (A.1), we evaluate the integral

$$
I_{i}=\int r^{\lambda} \rho(\boldsymbol{r}) \mathrm{d} \tau
$$

With the substitutions $x=(r-R(\vartheta)) / a$ and $S=R(\vartheta) / a$ it takes the form

$$
I_{\lambda}=\rho_{0} \int \mathrm{d} \Omega R^{\lambda+2}(\vartheta) a \int_{-S}^{\infty} \frac{(1+x / S)^{\lambda+2}}{1+\mathrm{e}^{x}} \mathrm{~d} x .
$$

We first perform the radial integration in (A.4). One easily sees that

$$
\begin{gathered}
\int_{-S}^{\infty} \frac{x^{2 m}}{1+\mathrm{e}^{x}} \mathrm{~d} x=\frac{1}{2 m+1} S^{2 m+1}+\int_{S}^{\infty} \frac{x^{2 m}}{1+\mathrm{e}^{x}} \mathrm{~d} x \quad(m=0,1,2, \ldots), \\
\int_{-S}^{\infty} \frac{x^{2 m-1}}{1+\mathrm{e}^{x}} \mathrm{~d} x=\frac{-S^{2 m}}{2 m}+\pi^{2 m}\left(2^{2 m-1}-1\right) \frac{\left|B_{2 m}\right|}{m}-\int_{S}^{\infty} \frac{x^{2 m-1}}{1+\mathrm{e}^{x}} \mathrm{~d} x \quad(m=1,2,3, \ldots) .
\end{gathered}
$$

Here we have used the definite integrals

$$
\int_{0}^{\infty} \frac{x^{2 m-1}}{1+\mathrm{e}^{x}} \mathrm{~d} x=\frac{\pi^{2 m}}{2 m}\left(2^{2 m-1}-1\right)\left|B_{2 m}\right| \quad(m=1,2, \ldots),
$$

with the Bernoulli numbers $B_{2}=\frac{1}{6}, B_{4}=-\frac{1}{30}, B_{6}=\frac{1}{42}, \ldots$ Evaluating the integrals on the right hand side of (A.5), we can take advantage of the fact that in most cases of practical application, the lower limit $S$ is much larger than unity. For a rare earth nucleus e.g., $R_{0} / a \approx 10$. For the nuclei and the deformations studied in this paper, we have always $R(\vartheta) / a=S>6$. We can therefore expand and approximate them by

$$
\int_{S}^{\infty} \frac{x^{n}}{1+\mathrm{e}^{x}} \mathrm{~d} x \approx \int_{S}^{\infty} x^{n} \mathrm{e}^{-x}\left(1-\mathrm{e}^{-x} \ldots\right) \mathrm{d} x=n ! \sum_{v=0}^{n} \mathrm{e}^{-s} \frac{s^{v}}{v !}+\mathrm{O}\left(\mathrm{e}^{-2 S}\right) \quad(S \gg 1) .
$$


Inserting eqs. (A.5), (A.7) into (A.4), one obtains after evaluating some algebraic sums

$$
\begin{aligned}
I_{\lambda}=\rho_{0} \int \mathrm{d} \Omega\{ & \frac{1}{\lambda+3} R^{\lambda+3}(\vartheta)+(\lambda+2) ! a^{\lambda+3} \mathrm{e}^{-S} \\
& \left.+(\pi a)^{2} R^{\lambda+1}(\vartheta) \sum_{n=0}^{\left[\frac{1}{2}(\lambda+1)\right]}\left(\begin{array}{c}
\lambda+2 \\
2 n+1
\end{array}\right)\left(\frac{\pi a}{R(\vartheta)}\right)^{2 n} \frac{\left|B_{2 n+2}\right|}{n+1}\left(2^{2 n+1}-1\right)\right\} .
\end{aligned}
$$

With the diffuseness parameter $d$ defined by

we can rewrite eq. (A.8) as

$$
d=\left(\pi a / R_{0}\right)^{2},
$$

$$
\begin{aligned}
I_{\lambda}= & \rho_{0} \int \mathrm{d} \Omega\left\{\frac{1}{\lambda+3} R^{\lambda+3}(\vartheta)+\frac{\lambda+2}{6} d R_{0}^{2} R^{\lambda+1}(\vartheta)\right. \\
& +\frac{\lambda(\lambda+1)(\lambda+2)}{6} \frac{7}{60} d^{2} R_{0}^{4} R^{\lambda-1}(\vartheta)+\ldots \\
& \left.+\frac{(\lambda+2) !}{\pi^{\lambda+3}} d^{\frac{1}{2}(\lambda+3)} R_{0}^{\lambda+3} \mathrm{e}^{-s}\right\},
\end{aligned}
$$

which is exact up to terms of $\mathrm{O}\left(\mathrm{e}^{-2 S}\right)$. We see that for values of $\lambda$ up to 4 , the last term in (A.10) is always several orders of magnitude smaller than the leading terms in the region of nuclei considered here. We can therefore neglect it completely (its relative contribution to $I_{0}$ is less than $\left.10^{-4}\right)$.

The angular integration in eq. (A.10) can now easily be performed using the orthogonality relations of the spherical harmonics in (A.2).

The volume conservation condition requires that

$$
I_{0}=\text { const. }=\rho_{0} \frac{4}{3} \pi R_{0}^{3} .
$$

If this is to be true independent of both deformation and diffuseness $d$, the value of $b_{0}$ in (A.2) has to be

$$
b_{0}=-\frac{1}{4 \pi}\left(\beta_{2}^{2}+\beta_{4}^{2}\right)-\frac{1}{3} d \text {. }
$$

Eq. (A.12) is exact up to terms of order $d^{3}, \beta_{i}^{2} d^{2}, \beta_{i}^{5}, \ldots$ (Thus the terms $\propto d^{2}$, $\beta_{i}^{2} d, \beta_{i}^{4}$ cancel identically!) The value $\rho_{0}$ of the central density is determined by setting $I_{0}$, eq. (A.11), equal to the particle number $Z$ or $N$.

For the mean square radius $\widetilde{R}^{2}$ we obtain

$$
\widetilde{R}^{2}=I_{2}=\frac{3}{5} Z R_{0}^{2}\left\{1+\frac{5}{4 \pi}\left(\beta_{2}^{2}+\beta_{4}^{2}\right)+\frac{3}{3} d+\mathrm{O}\left(d^{2}\right)\right\} .
$$


The multipole moments $\widetilde{Q}_{\lambda}$, defined by

$$
\tilde{Q}_{\lambda}=\sqrt{\frac{16 \pi}{2 \lambda+1}} \int r^{\lambda} Y_{\lambda, 0}(\vartheta) \rho(r) \mathrm{d} \tau,
$$

can now be evaluated using the formula (A.10) with an extra factor $Y_{\lambda}(\vartheta)$ under the angular integration. For the first two moments we obtain (for protons)

$$
\begin{aligned}
& \widetilde{Q}_{2}=\frac{3}{\sqrt{5 \pi}} Z R_{0}^{2}\left\{\beta_{2}\left(1+\frac{2}{3} d\right)+0.360 \beta_{2}^{2}+0.967 \beta_{2} \beta_{4}\right. \\
& \left.+0.328 \beta_{4}^{2}+0.023 \beta_{2}^{3}-0.021 \beta_{2}^{4}+0.499 \beta_{2}^{2} \beta_{4}\right\}, \\
& \widetilde{Q}_{4}=\frac{1}{\sqrt{\pi}} Z R_{0}^{4}\left\{\beta_{4}(1+3 d)+\left(1+\frac{5}{3} d\right)\left(0.725 \beta_{2}^{2}+0.983 \beta_{2} \beta_{4}\right.\right. \\
& \left.\left.+0.411 \beta_{4}^{2}\right)+0.416 \beta_{2}^{3}+1.656 \beta_{2}^{2} \beta_{4}+0.055 \beta_{2}^{4}\right\} .
\end{aligned}
$$

The first missing terms in eqs. (A.15), (A.16) are of order $d^{2} \beta_{i}, d^{2} \beta_{i} \beta_{j}$. (Note that the terms $\propto d \beta_{i} \beta_{j}$ have cancelled identically in eq. (A.15)!) Since $d$ is $\approx 0.06$ for actinide nuclei, we neglect the quadratic terms in $d$. The two eqs. (A.15) and (A.16) have been used in eqs. (11) and (12) for the case $d=0$.

From these results, one might conclude that the surface thickness clearly affects the valucs of the multipole moments. For $d \approx 0.06, \widetilde{Q}_{2}$ is increased by $\approx 4 \%$ and $\tilde{Q}_{4}$ by $\approx 20 \%$ compared to the value with $d=0$. However, there is some ambiguity in this result. First, it is not clear whether the dependence on $d$ has to be taken into account in the volume conservation condition (A.11). In the way this has been done above, $R(\vartheta)$ is for $d \neq 0$ not equal to the half-density radius, even for a spherical distribution. One might thus as well ${ }^{32}$ ) claim that $b_{0}=0$ for $\beta_{i}=0$. With this, onc would obtain

$$
\begin{gathered}
b_{0}=-\frac{1}{4 \pi}\left(\beta_{2}^{2}+\beta_{4}^{2}\right), \\
\widetilde{R}^{2}=\frac{3}{5} Z R_{0}^{2}\left\{1+\frac{5}{4 \pi}\left(\beta_{2}^{2}+\beta_{4}^{2}\right)+\frac{7}{3} d+\ldots\right\}, \\
\widetilde{Q}_{2}=\frac{3}{\sqrt{5 \pi}} Z R_{0}^{2}\left\{\beta_{2}(1+d)+0.360 \beta_{2}^{2}+\ldots\right\}, \\
\tilde{Q}_{4}=\frac{1}{\sqrt{\pi}} Z R_{0}^{4}\left\{\beta_{4}(1+4 d)+\left(1+\frac{7}{3} d\right)\left(0.725 \beta_{2}^{2}+\ldots\right)\right\} .
\end{gathered}
$$

With this alternative way to conserve the volume, even the lowest-order terms in $d$ have different factors in front.

Second, one can argue that the effective mean square radius, which is measured experimentally, depends on $d$. Thus, one might identify it with (following eq. (A.13))

$$
\widetilde{R}_{\text {eff }}^{2}=R_{0}^{2}\left(1+\frac{5}{3} d\right),
$$


and express the moment in terms of it. For $\tilde{Q}_{2}$ e.g., one obtains then

$$
\tilde{Q}_{2}=\frac{3}{\sqrt{5 \pi}} \mathrm{Z} \widetilde{R}_{\mathrm{eff}}^{2}\left\{\beta_{2}(1-d)+0.362 \beta_{2}^{2}+\ldots\right\} \text {. }
$$

The effect of $d$ on the moment $\widetilde{Q}_{4}$ would then be smaller than in the case (A.16). Still another expression is obtained by doing the same with eqs. (A.13.1), (A.15.1).

We are therefore left with an ambiguity in the dependence of the moments $\widetilde{Q}_{\lambda}$ on the diffuseness parameter $d$ even to lowest order. This reflects that the deformation parameters $\beta_{i}$ can be defined in different ways. They are not measurable quantities but can only be obtained indirectly through relations to the moments $Q_{\lambda}$. Thus the ambiguity discussed above means that they are not well defined to a percentage accuracy better than around $d$. Consequently, the dependence of $\widetilde{Q}_{\lambda}$ on $d$ is not unique and one does not lose anything using expressions like eqs. (11) and (12).

\section{References}

1) C. E. Bemis, Jr., F. K. McGowan, J. L. C. Ford, Jr., W. T. Milner, P. H. Stelson and R. L. Robinson, Phys. Rev. C8 (1973) 1466

2) H. J. Specht, J. Weber, E. Konecny and D. Heunemann, Phys. Lett. $41 B$ (1972) 43

3) R. Heffner, J. Pedersen and G. Sletten, private communication (1973)

4) V. Metag, private communication (1973)

5) U. Götz, H. C. Pauli, K. Alder and K. Junker, Nucl. Phys. A192 (1972) 1

6) V. M. Strutinsky, Nucl. Phys. A95 (1967) 420; A122 (1968) 1

7) M. Brack, J. Damgaard, A. S. Jensen, H. C. Pauli, V. M. Strutinsky and C. Y. Wong, Rev. Mod. Phys. 44 (1972) 320

8) P. Möller and S. G. Nilsson, Phys. Lett. 31 B (1970) 283

9) H. C. Pauli, T. Ledergerber and M. Brack, Phys. Lett. 34B (1971) 264

10) S. E. Larsson, I. Ragnarsson and S. G. Nilsson, Phys. Lett. 38B (1972) 269

11) U. Götz, H. C. Pauli, K. Alder and K. Junker, Phys. Lett. 38B (1972) 274

12) H. C. Pauli, Phys. Reports 7 (1973) 35

13) H. C. Pauli and T. Ledergerber, Nucl. Phys. A173 (1971) 398

14) D. Inglis, Phys. Rev. 96 (1954) 1059

15) P. Decowski, W. Grochulski, A. Marcinkowski, K. Siwek and Z. Wilhelmi, Nucl. Phys. A110 (1968) 129

16) A. S. Jensen and J. Damgaard, Nucl. Phys. A203 (1973) 578

17) S. G. Nilsson, C. F. Tsang, A. Sobiczewski, Z. Szymanski, S. Wychec, G. Gustafsson, I. L. Lamm, P. Möller and B. Nilsson, Nucl. Phys. A131 (1969) 1

18) A. Bohr and B. Mottelson, Nuclear structure, vol. 2 (Benjamin, New York) to be published

19) L. R. B. Elton, Introductory nuclear theory (Pitman, London, 1959)

20) W. D. Myers, Nucl. Phys. A145 (1970) 387

21) B. Nilsson, Nucl. Phys. A129 (1969) 445

22) P. Möller, J. R. Nix and S. G. Nilsson, Nucl. Phys. A229 (1974) 292

23) T. Grin, JETP (Sov. Phys.) 16 (1963) 1327

24) A. Bohr and B. Mottelson, Mat. Fys. Medd. Dan. Vid. Selsk. 30 (1955) no. 1

25) S. Belyaev, Mat. Fys. Medd. Dan. Vid. Selsk. 31 (1959) no. 11

26) G. Lüders, Z. Naturf. 15A (1960) 371

27) $\mathrm{Ph}$. Quentin, private communication (1974)

28) C. M. Lederer, J. M. Hollander and I. Perlman, Tables of isotopes (Wiley. New York, 1967)

29) J. Krumlinde, Nucl. Phys. A160 (1971) 471

30) A. Sobiczewski, S. Bjørnholm and K. Pomorski, Nucl. Phys. A202 (1973) 274

31) I. Hamamoto, Nordita preprint (1974)

32) L. W. Owen and G. R. Satchler, ORNL report 3525 (1963); Nucl. Phys. 51 (1964) 155 